Document downloaded from:

http://hdl.handle.net/10251/88387

This paper must be cited as:

Albero, J.; García Gómez, H. (2015). Doped graphenes in catalysis. Journal of Molecular Catalysis A: Chemical. 408:296-309. doi:10.1016/j.molcata.2015.06.011.

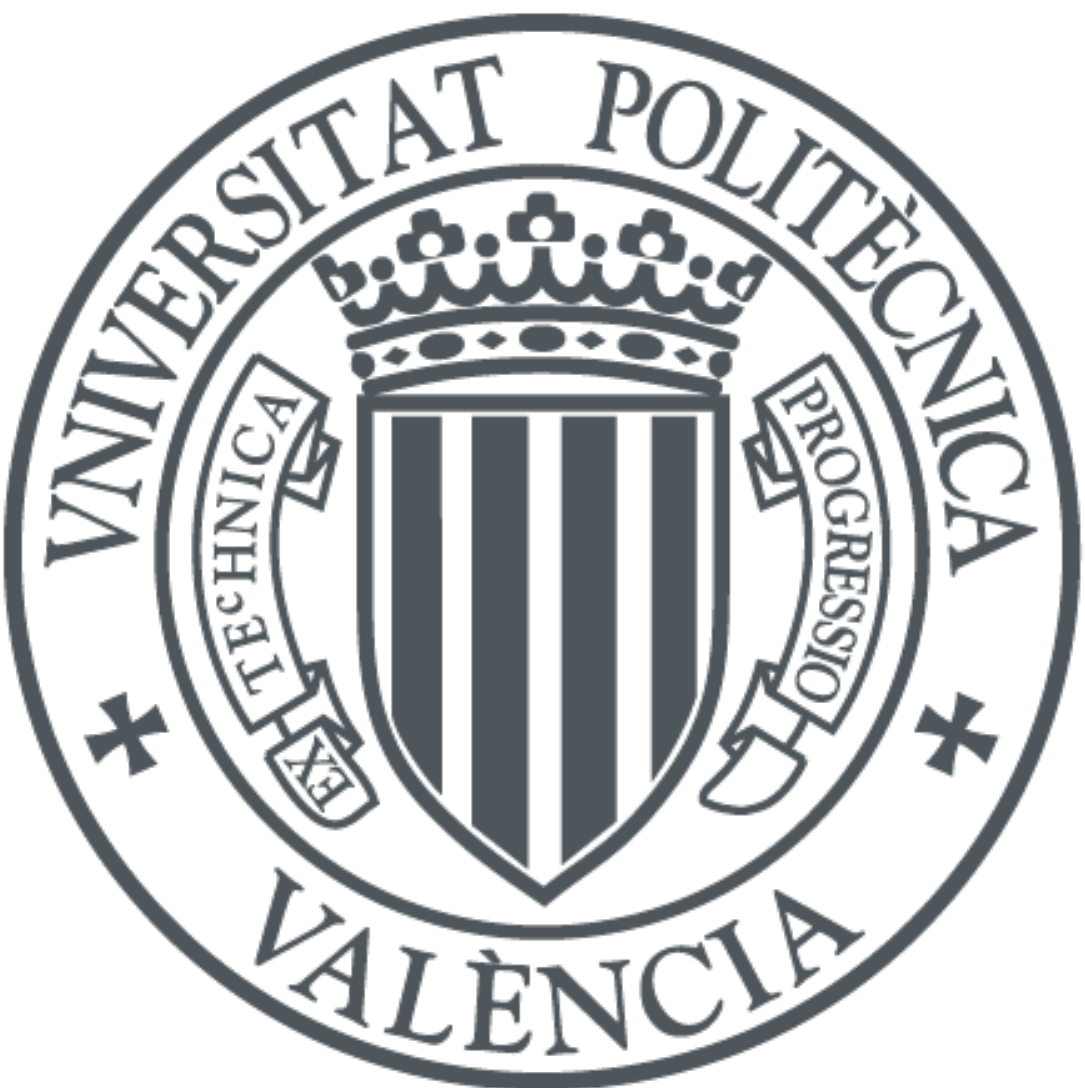

The final publication is available at

http://dx.doi.org/10.1016/j.molcata.2015.06.011

Copyright Elsevier

Additional Information 


\title{
Doped graphenes in catalysis
}

\author{
Josep Albero and Hermenegildo Garcia
}

Instituto Universitario de Tecnología Química CSIC-UPVC, Univ. Politecnica de Valencia, Av. De los Naranjos s/n, 46022 Valencia, Spain

\section{Abstract}

Due to the availability and easy preparation, graphenes are currently among intense investigation for various applications in chemistry including as metal-free catalyst. The presence in low percentage of heteroatoms on the graphene sheet has become a general strategy to modify the electron density, electrical conductivity and other properties of graphenes that can be reflected on the use of these materials in catalysis. In the present review we focus on those reports describing the use of doped graphenes as metal-free catalyst or as support of metal nanoparticles. After a brief introduction presenting the unique properties and features of graphenes and the reasons why they are suitable to be applicable in catalysis. Before the main body of the review describing common features and relevant examples of the use of doped graphenes in catalysis, the preparation method of doped graphenes and adequate characterization techniques providing important information, particularly with respect to the active site in catalysis, are briefly presented. Also general traits of graphenes of support of metal nanoparticles are commented. The final section summarizes the main conclusions of the review and provides our view future developments in the field. The purpose of the present review is not to provide an exhaustive account of all the existing literature, but rather to introduce the reader in the opportunities and advantages that doped graphenes offer with regard to the use either as metal free catalyst or support of metal nanoparticles. 


\section{Introduction and scope of the review}

Catalysis, either homogeneous or heterogeneous, is dominated by salts, complexes or materials containing transition metals, not infrequently noble and critical metals [1-9]. For the sake of rational use of resources and sustainability, there is a logical interest in replacing catalysts that contain $\mathrm{Pt}, \mathrm{Ir}, \mathrm{Rh}, \mathrm{Au}, \mathrm{Pd}, \mathrm{Ta}, \mathrm{Re}$ or rare earths by other type of catalysts [10-13]. The search for alternatives to noble and critical metals has led to explore the catalytic activity of other widely abundant metals such as $\mathrm{Fe}, \mathrm{Ni}, \mathrm{Cu}$, or $\mathrm{Al}$, but also to the development of the concept of carbocatalysis [14-17]. Carbocatalysis consists in the use as catalysts of materials containing exclusively or predominantly carbon as constitutive element. In contrast to organocatalysis in which well-defined organic molecules, either pure or supported on insoluble solids, are used to promote the chemical transformation, carbocatalysis are based on materials lacking a precise molecular structure and being considerably more complex than molecules. Materials require extensive characterization by a combination of different textural, spectroscopic and microscopic techniques in order to be characterized. Carbocatalysis has experienced a renewed growth since graphene $(G)$ and related one-atom thick 2D layered materials have become widely available. While active carbons have been used in catalysis as support of metal nanoparticles (NPs) since the early times of heterogeneous catalysis, their undefined structure and complexity has impeded their ample use as self-standing catalysts.

Since the eighties and after the discovery of fullerene and fulleroids, carbon allotropes have become common materials having a considerable potential in many areas from physics to chemistry and biology [18-20]. The discovery of fullerenes as carbon allotropes was subsequently followed by synthesis and characterization of carbon nanotubes (CNTs) either single or multi walled, and carbon nanohorns shifting from molecular carbon allotropes to materials with an indefinitely large number of carbon atoms $[21,22]$. However, the development of the full potential of CNTs in catalysis has been always hampered by the high cost of CNTs and the difficult preparation of sufficient quantities of these materials. Diamond NPs obtained either by milling of diamond particles or by explosive detonation have also appeared as promising carbon nanoforms in catalysis, but application of this material in catalysis is 
limited by the inertness of the diamond surface and, again, its availability and cost [23]. This situation has changed drastically in the case of G, particularly when obtained from graphite by exfoliation of graphite oxide (Scheme 1).

\section{Graphite Graphite oxide Graphene}

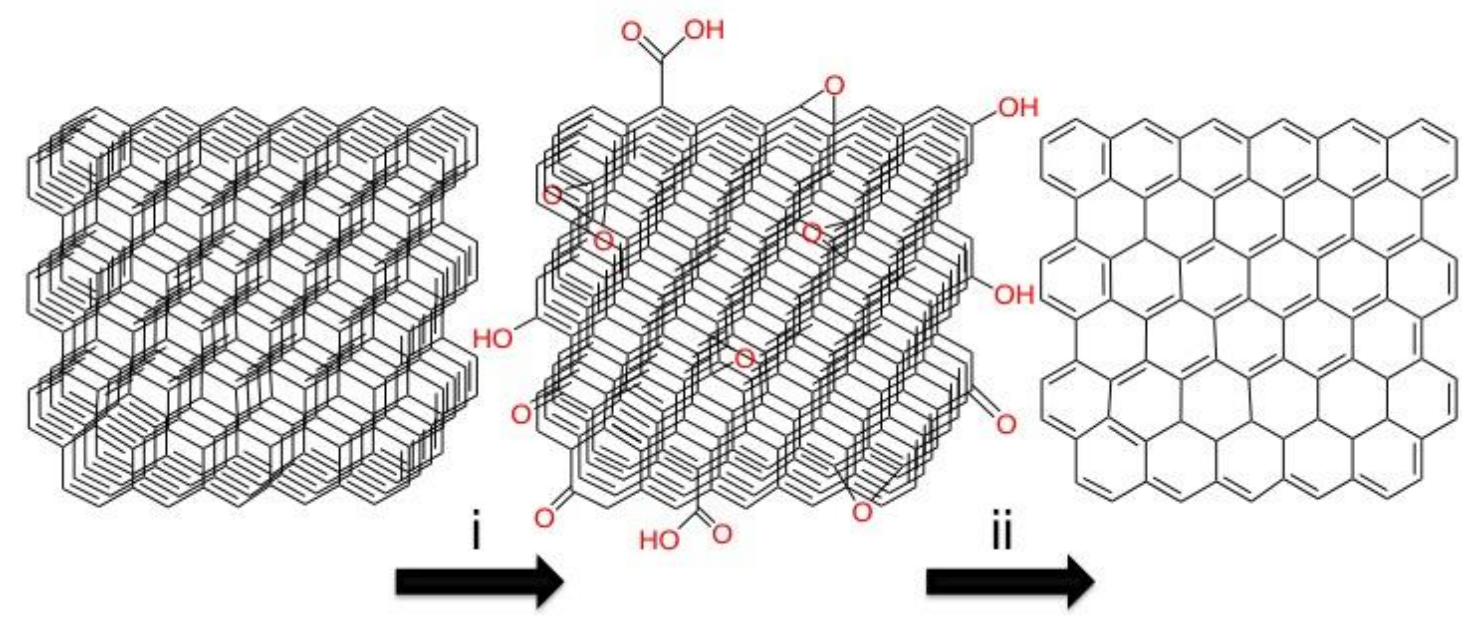

Scheme 1. Ideal structures of graphite, graphite oxide and $G$ and the synthetic sequence to obtain them. i) Deep chemical oxidation, and ii) exfoliation.

While for application in microelectronics highly conductive, defect-free $G$ is generally a prerequisite $[24,25]$, it has become apparent that other type of applications, and particularly as metal-free catalysts, the presence of defects can be highly desirable [26]. One particular case that is of special relevance in the present case is doping with heteroatoms, i.e. elements other than carbon, on the $\mathrm{G}$ sheet that can modify the properties of $G$ and introduce new effects, particularly active sites in catalysis (Scheme 2) [27-30].

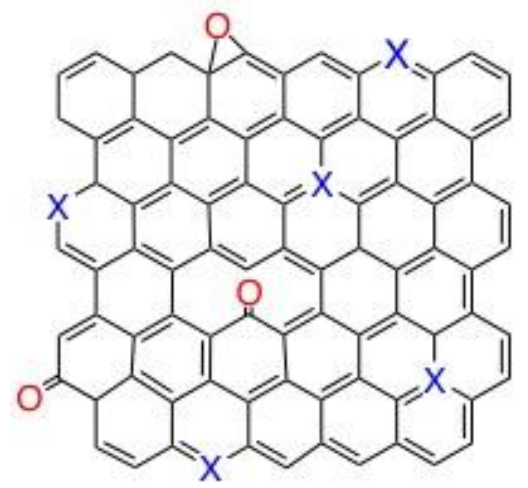


Scheme 2. Possible model of a doped graphene sheet with residual oxygenated groups and having heteroatoms $X$ in different positions, either peripheral, graphitic, at defects, etc.

In the present paper we will start summarizing the main properties of $G$ and related materials from the point of view of the use in catalysis, then, we will summarize preparation methods for doped Gs and suitable characterization techniques to determine the content and nature of the active sites. The main body of this paper would be a survey on the literature covering selected research articles in where doped Gs have been used either as metal-free catalyst or as support of metal or metal oxide nanoparticles. We will try to show the type of reactions that have been reported using doped Gs either as carbocatalysts as supports, making emphasis on the proposed role of the dopant element and the differences and advantages of these materials with respect to other alternative catalysts. Comments on how theoretical modeling can contribute to the progress and development of $G$ in catalysis will be illustrated by selected examples on the type of information that can be obtained from modeling and how this information can later be applied to develop new generations of more efficient metal-free carbon-based catalysts. The final section of this review provides a summary of the current situation and our views on future developments in the area. The intention of this review is not to cover exhaustively all the literature on Gs in catalysis, but to give a broad overview of the current state of the art, particularly in perspective with other alternative catalyst, and to highlight the potential that $G$ offer to be applied in other reaction types. The reader is referred to the existing reviews in the literature for a compressive coverage on the use of $G$ and related materials as catalysts, electrocatalysts and photocatalysts [31-34]. Especially we note the recent contribution by Q-W. Chen and H. Garcia covering in larger detail the catalytic activity of $G$ materials $[33,35]$. It should be commented that due to the electrical conductivity Gs had been among the most studied electrodes replacing Pt and other novel metals. Similarly, the semiconducting properties of some Gs make them suitable photocatalyst for degradation of pollutants in water or generation of hydrogen or methane from water or $\mathrm{CO}_{2}$, respectively, upon light irradiation. The fact that doped Gs are now under the spotlight with respect to their use in catalysis can be 
easily proved by the fact that most of the core references in the present manuscript have appear in 2010 or after and their number is exponentially growing.

\section{Unique features of $\mathbf{G}$ and related materials with regard to their use in catalysis.}

Heterogeneous catalysis, characterized by the existence of a surface that defines two different phases is always interested in developing materials with larger surface area. In a certain way heterogeneous catalysis is always an interfacial phenomenon occurring on the surface that limits two different phases. In this regard the importance of $\mathrm{G}$ and related materials derives from the consideration that being one atom thick layered materials they represent the physical limit of an ideal surface. In other words, on a simplistic way, $G$ can be considered like a surface in where catalysis would take place with all the atoms of the material being present on the surface. In normal solid catalysts, only the small proportion of those atoms located on the external surface of the particle can exhibit catalytic activity, while the predominant proportion of atoms in the interior of the particle are catalytic irrelevant. On the contrary, all the atoms present in $\mathrm{G}$ will be catalytically relevant since they will be exposed to substrates and reagents. This miniaturization of the surface occurring in Gs and related materials represent the maximum efficiency in the use of atoms for catalysis.

A second feature that is highly important for the use of Gs in catalysis is the strong adsorption of substrates or reagents on $G$ as consequence of the sterically favorable interaction with the extended $\pi$ orbital on $G$ (see Scheme 3). Simple models of $G$ sheets consider the presence of a fully accessible extended $\pi$ cloud above and below the carbon atoms that can strongly interact with substrates that approach the surface. In the case of CNTs having a rolled G wall, formation of strong supramolecular association complexes between CNTs and polycyclic aromatic molecules or metal complexes has been reported [36-39] and similar strong $\pi-\pi$ interactions must occur even more favorably on flat and flexible Gs. Adsorption is the initial step in any heterogeneous catalysis mechanism and in the case of $\mathrm{G}$ adsorption is favored by the geometry and the electronic configuration on the surface. The presence of heteroatoms should generate a distortion of this $\pi$ cloud and may introduce empty or 
additional orbitals. Therefore, doping can increase adsorption of certain substrates either by donating electrons, for instance in the case of $\mathrm{N}$ doping, or by accepting electrons as in the case of B doping, from substrates to the dopant atom in G.

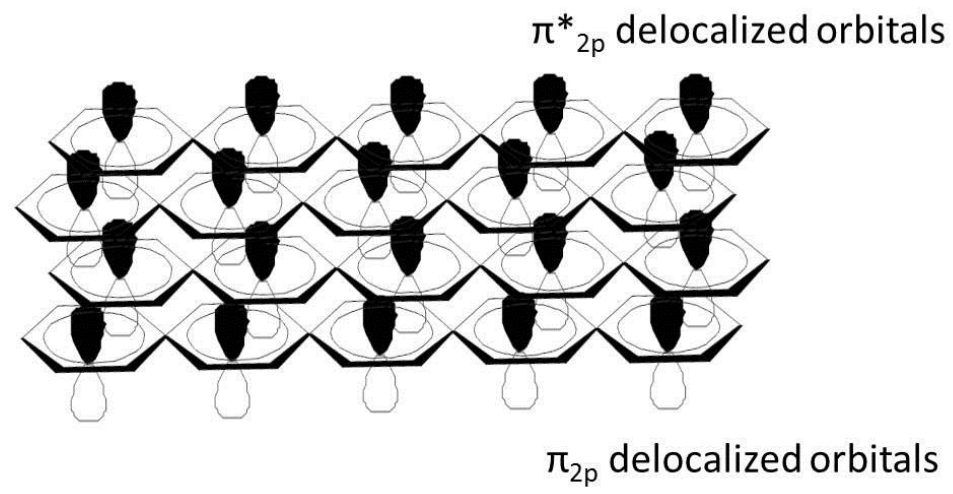

Scheme 3. Pictorial representation of the extended $\pi$ orbitals in a $\mathrm{G}$ sheet.

Besides large surface area and strong adsorption another key feature of Gs is the possibility to incorporate on the sheet active centers [33]. These active centers are generally associated to the presence of defects or heteroatom doping on the sheet, ideal highly-conductive $G$ being in most cases devoid of any catalytic activity. As an important example of these defects, carbon atom vacancies will leave dangling bonds in the neighbor carbon atoms around the vacancy that may intervene in the reaction mechanism completing their valences during certain steps in the mechanism. In this sense, holes in the $\mathrm{G}$ sheet can be clearly seen in some high resolution TEM images of some $\mathrm{G}$ samples and they have been proposed to be catalytically relevant in aerobic oxidations of benzyl amines, since they may possibly interact with molecular oxygen [40]. Another type of defects, related also with the presence of heteroatoms, is oxygenated functional groups such as carboxylic acids, hydroxyl groups, epoxides and quinone-like carbonyl groups (see Scheme 4). Organic chemistry shows that all these oxygenated functional groups can participate in a large variety of processes. Not surprisingly, GO having high oxygen content (frequently above $40 \%$ ) has been frequently reported to act as catalyst for aerobic oxidations of benzylic alcohols and alkene polymerisation, while on the other extreme high electronic quality $\mathrm{G}$ as catalyst has not been used as catalyst. 
The use of $G$ in catalysis is surely inspired by previous reports using other carbon allotropes as catalysts. There are some examples using CNT catalysis in which selective protection of each of the three predominant types of oxygenated functional groups (carboxylic acid, hydroxyl groups and carbonyls) has allowed to propose that the catalytic activity should be attributed exclusively to one of these sites. This is the case of the oxidative dehydrogenation of light alkanes into alkenes promoted by CNTs for which evidence comparing the catalytic activity of pristine CNT with that of modified CNTs by protecting specifically an oxygenated functional group strongly supports that quinone-like structures are the active sites in this process [41]. However, a major difference between CNTs and GO is the high $\mathrm{O}$ content of the latter, with a large population of epoxide groups and this could determine that the active sites of GO for this process could be different than those of CNTs, even for the same process. In fact, it has been proposed that the oxidative dehydrogenation of light alkanes catalyzed are promoted by epoxides in GO rather than quinones as in the case of CNTs [42]. Thus, it is the methodology rather than the conclusions what has to be applied when transferring the knowledge from other allotropic forms to catalysis by Gs.

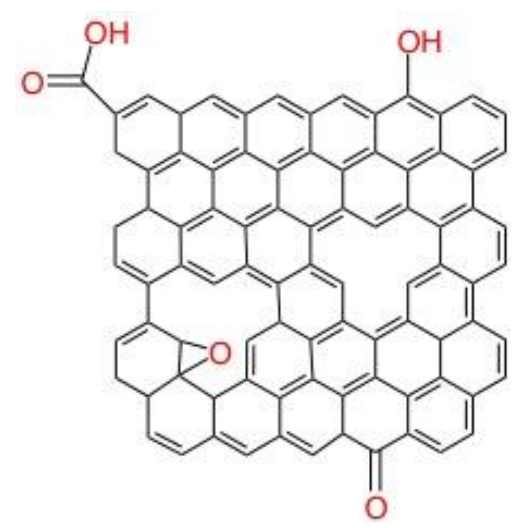

Scheme 4. Representation of a G sheet with possible oxygenated defects and atom vacancies.

More related to the focus of the present review is the activity of dopant elements as active sites. Experimental evidence of the catalytic activity of the dopant atoms should be obtained by comparing the catalytic activity of undoped and doped Gs at different loading or Gs doped with different hetreoatoms. To determine the role of a given dopant element in catalysis, it would be very convenient if a systematic 
series of doped Gs at various loadings are prepared and the kinetic measurements correlated with the heteroatom loading. However, preparation of these samples is not always possible and the area is still needed of more flexible and convenient preparation procedures to obtain doped G. Besides sample preparation, there is in general a paucity of detailed understanding of how dopant elements can intervene in the reaction mechanism. Computational chemistry will be certainly applied massively in this field in the near future, as it is the tendency in heterogeneous catalysis. In one of the still limited examples, it has been found that $\mathrm{N}$ atoms on $\mathrm{N}$-doped $\mathrm{G}$ can decompose hydrogen peroxide to oxygen or water [43] by forming a $\mathrm{N}$-hydroxyl intermediate.

The most important remark of this introductory section is the emphasis in the relationship between $G$ catalytic activity and the presence of defects. While highly-conductive, high-quality $G$ can be ideal for application of these materials in microelectronics, for the purpose of exploiting the potential of $\mathrm{G}$ materials in catalysis, the presence of defects in the broad sense is necessary. One key concept in this area is that catalytically active sites are associated to defects in the "ideal" structure of the hexagonal array of $\mathrm{sp}^{2}$ carbon atoms. This comment raises the issue, frequently ignored for outsiders in catalysis, that the ideal $\mathrm{G}$ material for electronics is surely not be the ideal one for other applications, particularly for catalysis. Furthermore, in a similar way, a G material having high catalytic activity for a certain reaction may not be the most adequate catalyst for other reaction type. Thus, caution should be taken when considering electrical conductivity as the only criteria to assess quality of $\mathrm{G}$ for applications not strictly related with microelectronics. One clear example of the above is the know photocatalytic activity of $\mathrm{GO}$, while $\mathrm{G}$ is photocatalytically inactive due to its zero band gap [44]. It is, however, true that at the present state of the art, GO, reduced $\mathrm{GO}(\mathrm{rGO})$ and doped $\mathrm{Gs}$ are the $\mathrm{G}$ materials that have exhibited so far the widest catalytic activity.

\section{Preparation of doped Gs.}


One of the possibilities to incorporate an active site on a $G$ sheet is to replace one carbon atom by another element, leading to doped G. The most widely used dopant elements are $\mathrm{N}$ and $\mathrm{B}$, but doping with $\mathrm{P}, \mathrm{S}$ have also been reported [27-30] . It can be in a certain way also considered that $G$ samples with low oxygen content such as rGO corresponds also to a case of oxygen-doped G. It would be important in the area, and this is a task that surely will develop in the near future, that Gs doped with other non-metallic and metallic elements will be prepared and their activity tested as catalyst.

Besides doping, an additional possibility would be to have simultaneously more than one dopant element, this resulting in co-doped $G$ materials. Certainly the combination of dopant heteroatoms having full and empty orbitals will be of large potential application in catalysis, in view that the combination of Lewis basic and acid sites of low to moderate strength in solid catalysts is able to promote a large number of condensation reactions [45-47].

For doped Gs the key parameters with impact in catalysis are the loading of the dopant element and its distribution among various different types according to their bonds. The presence of one dopant element introduces electronic and geometrical stress on the $\mathrm{G}$ sheet, causing around the dopant element a remarkable deviation from the local electronic density, bond angles and planarity of the ideal G. Among other considerations dopant element can introduce Lewis acid or basic sites depending on the number of electrons or empty orbitals introduced by the heteroatom, but also can act accepting or donating electronic density in redox processes. Theoretical calculations have shown that, working on simple models, the presence of heteroatoms generates a gap between the occupied and unoccupied frontier orbitals and accordingly doped $\mathrm{G}$ should exhibit a behavior as semiconductor in contrast to the conductive properties to the ideal G [48].

There are several possibilities to prepare doped $G$ (Scheme 5). For the $G$ synthesis based on chemical vapor deposition (CVD) using hot metal surfaces as template in the formation of the G film, the gas phase should contain a precursor containing the heteroatom to be incorporated on G. For instance, the presence of 
ammonia in combination with methane or other hydrocarbon in a hydrogen atmosphere has been reported as a way to dope with nitrogen atoms during the formation of G by CVD [49].

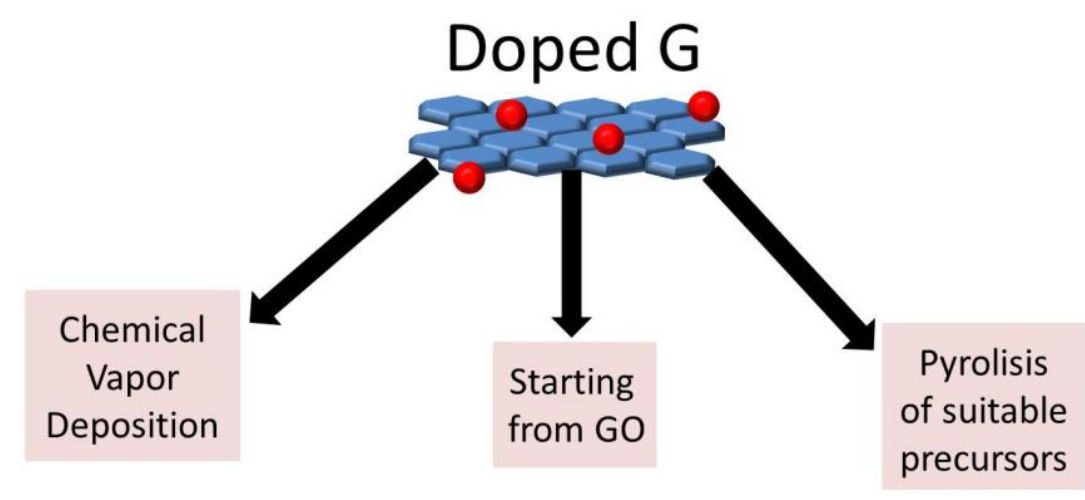

Scheme 5. Some important preparation methods to obtain doped G.

Another procedure for the preparation of doped G starts with GO that reacts, typically in water or liquid phase, with a suitable reagent containing the dopant element, for instance $\mathrm{NH}_{3}$ or $\mathrm{HS}^{-}$. Epoxy, hydroxyl and carbonyl groups present on $\mathrm{GO}$ can react with these precursors by substitution, condensation or nucleophilic attack leading to a functionalized $\mathrm{GO}$ in which the dopant element has been bonded to $\mathrm{C}$. Subsequent reduction of GO with partial reconstitution of the $\mathrm{G}$ structure can result in the incorporation of some heteroatoms. This procedure is very convenient considering the easy availability of GO, its solubility in water and its large chemical reactivity with a large variety of nucleophilic reagents. In principle the loading levels that can be achieved from GO can be very large, considering the proportion of functional groups. However, more information about the type of bonds of the heteroatom on Gs obtained from GO in comparison with the materials prepared by CVD has to be studied trying to delineate coincidences and differences.

Another procedure for the preparation of doped $G$ that has been reported by our group consists on the pyrolysis under inert atmosphere of a natural biopolymer that is able to form doped $G$ that has been modified by derivatisation with units containing the required heteroatoms. For instance, chitosan, a polysaccharide of glucosamine that contains about $11 \mathrm{wt} \% \mathrm{~N}$ can act simultaneously as source of carbon and nitrogen, rendering upon pyrolysis at temperatures above $900{ }^{\circ} \mathrm{C}$ under inert 
atmosphere N-doped G [50]. Similarly, $\Lambda$-carragenan containing a high proportion of sulfated galactose affords upon pyrolysis S-doped G [51]. The important parameters to be control in the pyrolysis are the temperature and time of the process. It has been found that in this procedure, the electrical conductivity of $G$ increases along the pyrolysis temperature as consequence of the decrease in the number of defects on the $\mathrm{G}$ sheet. Also the proportion of the heteroatom decreases upon increasing the pyrolysis temperature in the range from $900-1500{ }^{\circ} \mathrm{C}$. This general relationship between pyrolysis temperature and dopant loading is a reflection of the healing of the $\mathrm{G}$ sheet observed as the pyrolysis temperature increase.

A further procedure based on the pyrolysis of biopolymers consists in submitting to this treatment modified derivatives. For instance alginate, a polysaccharide of mannuronic and guluronic acids, can be esterified with boric or phosphoric acids [52]. Hydroxyl groups of sugars tend to from esters with inorganic and carboxylic acids [53]. Pyrolysis of these modified esters of inorganic acids result in the formation of Gs containing the heteroatom present in the inorganic ester, for instance, $B, S$ and $P$. Furthermore if chitosan, already containing $N$, is modified with boric acid to form the corresponding borate ester, then pyrolysis of this modified chitosan results in the formation of a N and B co-doped G [47].

\section{Characterization techniques.}

As commented earlier one important issue from the point of view of characterization of active sites in doped Gs is the nature, content and loading of the dopant element. XPS as quantitative analytical technique provides information about the presence of the dopant element and its proportion. In addition, XPS provides also valuable information on the types of different dopant elements according to the bonding and surroundings on the structure. For instance, deconvolution of the broad N1s peak in XPS for N-doped G into individual components has led to the conclusion that $\mathrm{N}$-doped $\mathrm{G}$ may contain up to three types of $\mathrm{N}$ atoms that can be assigned based on their binding energy values into graphenic, pyridinic and pyrrolic nitrogens (see Scheme 6) [54]. Pure organic molecules having a single type of heteroatom exhibit narrow peaks in XPS with less than $2 \mathrm{eV}$ width with specific binding energy values. In 
contrast, the experimental peaks in doped Gs are much broader, sometime expanding tens of eV. The broadening is due to the overlap of different types of atoms, whose assignment can be made by comparison with the binding energy values of this element in pure molecules, as commented in the case of nitrogen. The presence of various types of heteroatoms in doped graphene is highly relevant from the point of view of their catalytic activity and, in this regard, the information obtained by XPS is extremely useful. As the chemical reactivity of pyrrol or pyridine is remarkable different, it is also expected that when incorporated on the $\mathrm{G}$ sheet the different population of $\mathrm{N}$ can exhibit different activity. As an example of the methodology that can be followed to determine the catalytic activity of each different type of dopant atom, the catalytic activity of a series of $\mathrm{N}$-doped Gs for nitroaromatic reduction with sodium borohydride has been correlated exclusively with the concentration of graphenic $\mathrm{N}$ atoms, rather than with the total $\mathrm{N}$ content of $\mathrm{G}[55,56]$. Accordingly, combustion chemical analysis that gives the total content of heteroatom is considerably less informative, or even can be misleading, with respect to the information that can be obtained by careful consideration of the information contained in XPS.

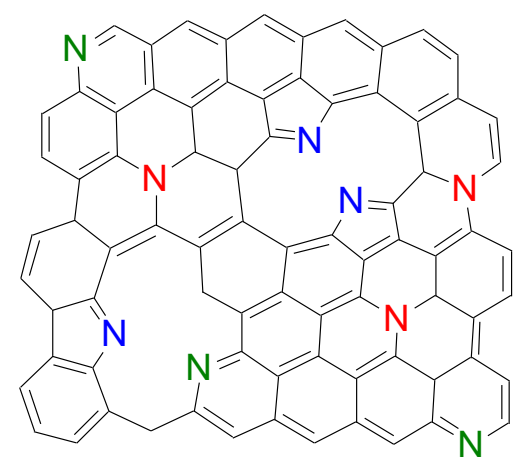

Scheme 6. Idealized model of $\mathrm{N}$-doped $\mathrm{G}$ including graphenic nitrogen atoms (red), pyridinic nitrogen atoms (green) and pyrrolic nitrogen atoms (blue).

As just commented, the presence of heteroatoms and its content can be quantified by combustion chemical analysis and XPS. These two analytical techniques have to be combined with the information of at least three other characterization techniques that are general to assess the morphology and structure of Gs, i.e. Raman spectroscopy, TEM and AFM. 
In Raman spectroscopy the presence and relative intensity of the $G$ band associated to graphitic structures with respect to the $D$ band due to the presence of $\mathrm{sp}^{3}$ carbons and defects gives quantitative indication of the quality and density of defects on the $\mathrm{G}$ sheet. It should be commented that doping can be considered as a defect and this is generally reflected in a high relative intensity of the $D$ band. TEM should show the 2D morphology characteristic Gs and the presence of wrinkles indicative of the flexibility of $\mathrm{G}$ sheets as consequence of the single layer structure (see Figure 1). High resolution TEM should reveal the structural ordering of atoms in hexagonal arrangement characteristic of G. Selected area electron diffraction should also show an electron diffraction pattern revealing the ordering and strict hexagonal arrangement at the nanometric dimensions expected for these materials.

Although Raman can provide also some indication of the single or few layer configuration of $\mathrm{G}$ by analyzing the position and intensity of the $2 \mathrm{D}$ bands at about $2500 \mathrm{~cm}^{-1}$, the safest way to determine the single layer configuration of $\mathrm{G}$ sheet is by AFM, casting a drop of the $G$ suspension on atomically flat surfaces of mica or other supports. AFM has subnanometric vertical resolution and by measuring the thickness of a $G$ sheet the number of layers of one particle can be determined. Special care should be paid when reaching conclusions based on microscopy techniques, since different parts of the sample should be studied providing statistical count of different particles all over the sample and never just based on a single image in order to obtain an adequate understanding of the sample under study with sufficient degree of confidence.

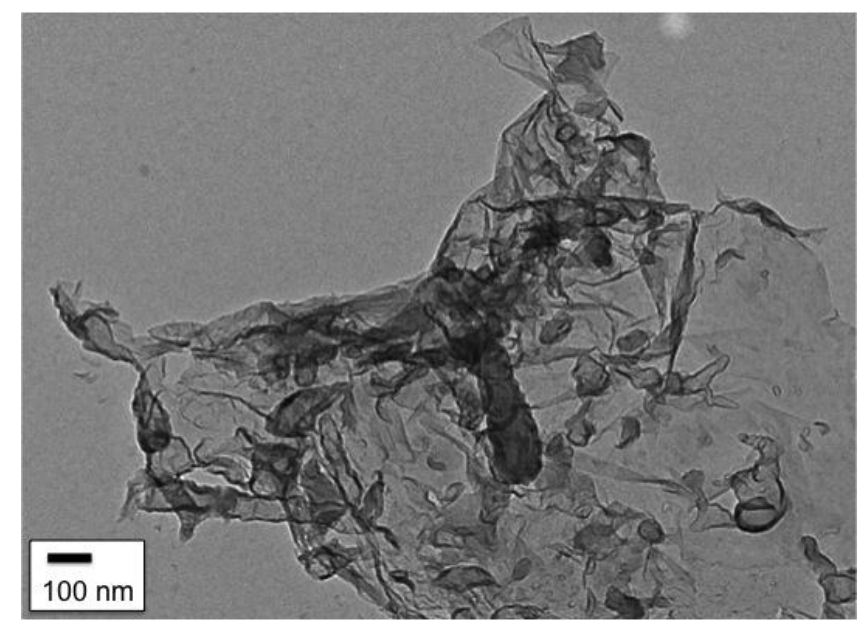


Figure 1. TEM image of a $G$ sheet with typical wrinkles due to the flexibility of the sheets. The presence of small debris on the material can also be observed.

Thermogravimetric techniques are also highly valuable for the application of $\mathrm{G}$ in catalysis. These techniques can report on the interaction of doped Gs with acid or base probe molecules or oxidizing and reducing reagents. In this way, the acid sites of a doped $\mathrm{G}$ sample can be reacted with ammonia in the gas phase and then after cleaning the surface by removing physisorbed ammonia, thermoprogramed desorption (TPD) can determine the amount of ammonia that is interacting with acid sites. Similar studies can be made with $\mathrm{CO}_{2}$ as a probe of possible basic sites present of doped $\mathrm{G}$. Also hydrogen TPD can be used to determine the ability of doped $G$ to become reduced by hydrogen. In these gravimetric techniques an alternative to expose $\mathrm{G}$ to a single dose of the probe molecule at a given temperature would be to expose the $G$ sample to a hydrogen stream at increasing temperatures while determining the amount of hydrogen that is retained by $\mathrm{G}$ as a function of the temperature. This could be particularly useful in those cases in which the active sites of $G$ do not react with the probe at room temperature, but they can be activated thermally. These conditions can be more alike to those used during the reaction, such as in oxidations or hydrogenations. In this way the density of sites that can be oxidized/reduced as a function of the temperature can be determined for different samples of doped G.

\section{Theory for understanding the activity of doped Gs.}

Heterogeneous catalysis has benefited enormously from the combination of powerful characterization techniques and theoretical calculations. While initial development of solid catalysts was based on trial and error tests, it has become evident that understanding of the catalytic activity of a material requires the combination of information provided by experimental techniques to determine the number and structure of active sites with rationalisation from quantum chemical calculations providing a conceptual framework to rationalize the experimental data. This general multidisciplinar strategy to study the catalytic activity of materials has to be necessarily also applied to the case of doped Gs. As we have commented in the previous section, experimental correlations between kinetic data and the content and 
distribution of dopant elements is considered firm evidence in support of the involvement of these heterotatoms in the reaction mechanism. However, reasonable mechanistic proposals had to be accompanied by theoretical calculations supporting the feasibility of the elementary steps of the proposal with indication of the energy changes in each individual process and indication of the rate determining step and structure of the transition state.

Due to the fact that the use of Gs in catalysis is a recent research field, there is an urgent need for theoretical studies providing rationalization of the catalytic activity introduced by the dopant elements. However, considering the simplicity and the monolayer morphology, Gs offer considerable opportunities for application of quantum mechanics. As an example, the interaction of hydrogen with doped $G$ has been studied theoretically. Activation of molecular hydrogen by $G$ can be of importance in the reversible transformation of $G$ into graphane, in the context of hydrogen storage and also in the use of $G$ as hydrogenation catalyst [57]. DFT calculations of $\mathrm{B}, \mathrm{N}$ or $\mathrm{O}$ doped $\mathrm{G}$ predict that when these elements are located at the zig-zag edges they should be able to promote dissociative adsorption of hydrogen [58]. In contrast, interaction of hydrogen with armchair edges in much less favorable due to the mismatch between $\mathrm{H}-\mathrm{H}$ bond length and the distance of the atoms at the periphery. Moreover, these calculations strongly suggest that $B$ is more suited for this potentially important process than $\mathrm{N}$ or $\mathrm{O}$. It would be convenient that these predictions based on calculations can be used as starting point in the design and preparation of suitable catalysts for dissociative hydrogen adsorption that could lead to the development of materials for reversible hydrogen adsorption-desorption or as hydrogenation catalysts [59]. Similarly, other DFT calculations have shown that $\mathrm{N}$ atoms can act as catalytic sites for the decomposition of hydrogen peroxide into oxygen (Scheme 7) $[43,60]$. In this case $N$-hydroxyl species appear to be the intermediates in the decomposition process rather than free hydroxyl radicals. 


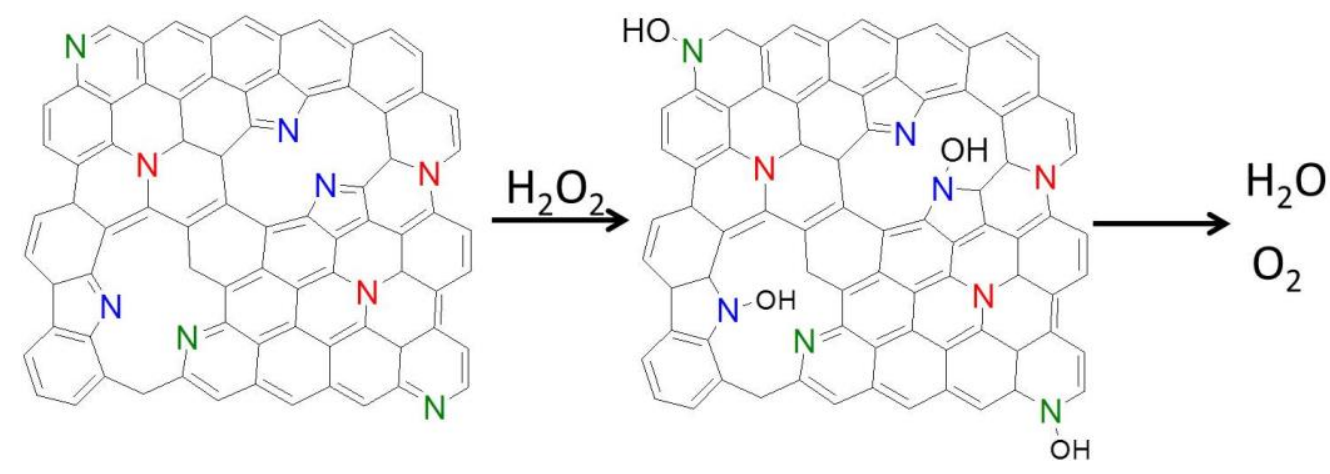

Scheme 7. Catalytic hydrogen peroxide decomposition mediated a N-doped G.

\section{Doped Gs as supports of metal NPs.}

Besides the use of $G$ and doped $G$ s as catalysts, these materials are extremely suitable as support of metal NPs that are really the relevant catalytic species (see Scheme 8). For application of a material as support of metal NPs, one crucial point is the interaction between the surface of the material and the supported NP. Strong metal-support interactions can lead to stabilization of the supported NP, but they can also modulate the catalytic activity. Also in this application of $G$, the presence of dopant elements on $\mathrm{G}$ should play an important role as predicted by theory.

In some of these studies, for the sake of simplicity the NP-support interaction has been estimated by calculating just the energy of interaction between a single metal atom and $G[61,62]$. Although this is a very much simplified model of the interaction between Gs and metal NPs, the results obtained by determining the interaction of a single metal atom can indicate a trend that could be applicable for NPs. Thus, for the case of N-doped G, it was found that the interaction energy between $\mathrm{Au}$ or other metallic elements should be higher, indicating the most probably location of the metallic element, on top of $\mathrm{N}$ atoms present in $\mathrm{G}$ rather than on the carbons. The interaction between dopant $\mathrm{N}$ atoms with Au metal atoms, being much stronger than that between $\mathrm{Au}$ atom and carbon, is presumed to contribute also to the stabilization of Au NPs on N-doped G during the catalytic cycle, with respect to undoped G. It is clear that this type of theoretical calculations, whose number is quite limited at the present, can be a very powerful tool when combined with catalytic data and kinetic studies to understand the role of $G$ as support of metal NPs. Using single atoms rather than NPs should make calculations much more doable at low computational cost and 
should be more likely to predict the behavior of NPs on doped Gs than modeling of more complex heterogeneous solid catalysts constituted by different crystal phases having thickness and requiring a much larger number of atoms in the slab to provide a meaningful result.

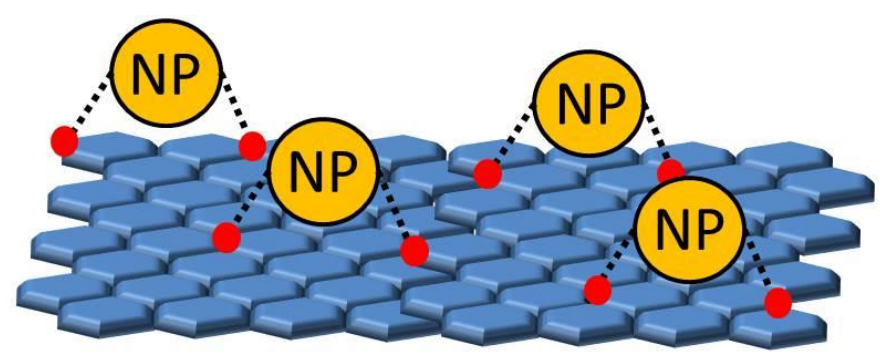

Scheme 8. Pictorial representation of metal NP supported on doped G material.

\section{Reactions catalyzed by doped Gs.}

Since the field of doped Gs as catalysts can be considered that has started in 2010, the examples that have been reported are still limited in comparison with the number that is expected in the next few years. Rather than providing an exhaustive account of the existing literature, the aim of the present article is to give an overview to show the opportunities and advantages that doped Gs offer in catalysis due to their 2D morphology, large surface area, dispersability and other features of $G$ that make this material unique among the different allotropic carbon nanoforms or other nanomaterials. The selected examples are trying to cover a reaction range as large as possible, although it is clear that it should be expected that the breadth of the reactions that will be promoted by doped $\mathrm{G}$ as catalyst with expand to many other reaction types.

Doped Gs have been used as catalysts for various oxidation reactions, either using molecular oxygen as oxidant or other oxidizing reagents including organic or inorganic peroxides. Of particular interest is the general activity of $\mathrm{G}$ materials to activate molecular oxygen through autooxidation processes. This ability of Gs to generate $\mathrm{C}$-centered radicals is probably to be related to the presence of dangling bonds at vacancies on the G sheet. GO and graphite oxide is a well-known material for this type of oxidations, but also doped Gs. 
Specifically, it has been found that $\mathrm{N}$ atoms are the active sites in the aerobic oxidation of benzyl alcohol using N-doped r-GO as catalyst (see Scheme 9). The reaction can be conveniently carried out in aqueous media using oxygen at atmospheric pressure. These experimental conditions minimize explosion hazards that are inherent to the use of pure oxygen with most of organic compounds [63]. Oxidations of primary alcohols to carbonylic compounds is a reaction type widely used in organic synthesis and for the production of fine chemicals used as fragrances and commodities. Typically this reaction can be catalyzed by transition metal catalysts, particularly by supported Pt or Au catalysts. The possibility to use a metal-free catalyst, such as N-doped $\mathrm{G}$ is a step forward in heterogeneous catalysis.

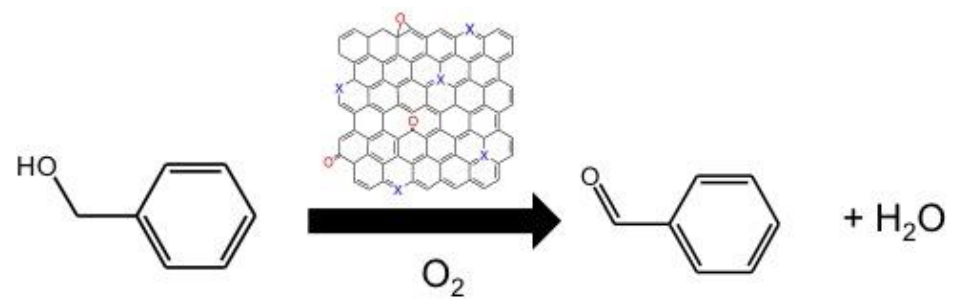

Scheme 9. Oxidation of benzyl alcohol to benzaldehyde by molecular oxygen promoted by $\mathrm{N}$-doped r-GO.

Co-doping of $B$ and $N$ renders a $[B, N] G$ sample that has been found the most efficient doped $G$ catalyst among the doped $G$ tested for the aerobic oxidation of benzyl hydrocarbons, cyclooctane and styrene [47]. Based on the comparison of catalytic activity of $[B, N] G$ with that of $[B] G$ and $[N] G, G O$ and $r G O$, it was proposed that $\mathrm{B}$ and $\mathrm{N}$ co-doping produces a synergism of the catalytic activity. As described earlier, all of these doped $G$ materials can be obtained conveniently by pyrolysis of widely available natural biopolymers (alginate or chitosan) for $G$ and [N]G, respectively, without or after being modified with boric acid that provides B for codoping. Thus, chitosan containing $\mathrm{C}$ and $\mathrm{N}$ can be esterified with boric acid to render a carbon residue that after sonication forms $[B, N] G$. XPS analysis of the exfoliated carbon residue provides strong evidence supporting the presence of the dopant elements, the percentage of these dopant elements in the material and, very importantly, it can provide information about the distribution of each dopant element into different families depending on the nature of the bonds and position of the 
element. In the present case of pyrolysis of chitosan borate, XPS analysis of $[B, N] G$ shows the presence of a single population of B atoms whose binding energy value correspond to that of $B$ atoms bonded to carbon. In contrast, deconvolution of the $\mathrm{N}$ 1s peak into three components shows that $\mathrm{N}$ atoms are distributed in this material in three main families corresponding to graphenic $\mathrm{N}$, pyridine and pyrrolic $\mathrm{N}$ atoms, in agreement with previous reports on the preparation of $\mathrm{N}$-doped $\mathrm{G}$ by other procedures. The synergy between $\mathrm{B}$ and $\mathrm{N}$ in the catalytic activity seems to suggest that activation of molecular oxygen is more favorable when two dopant elements are present. This synergism resembles the one that has been previously claimed for other solid catalysts, for instance, between acid and basic sites [47]. Thus, it has been found that the simultaneous presence of acid and basic sites of moderate strength can activate more efficiently the condensation of carbonyl groups than just other solid catalysts containing independently acid or basic sites even with higher strength. B and $\mathrm{N}$ atoms on the $\mathrm{G}$ sheet should behave as Lewis acid and basic sites, respectively, and the simultaneous presence of both can activate substrates and reagents by polarizing them and stabilizing polar intermediates. Using $[B, N] G$ as catalyst tetraline and another benzylic hydrocarbons can be oxidized to the corresponding ol/one mixture with high selectivity at high conversions in the absence of solvent [47]. The reaction mechanism seems to be auto oxidation of the benzylic position with the intermediacy of benzyl radicals reacting with molecular oxygen, since the reaction can be quenched by radical trapping agents. Cyclooctane can also similarly be oxidized to the ol/one mixture.

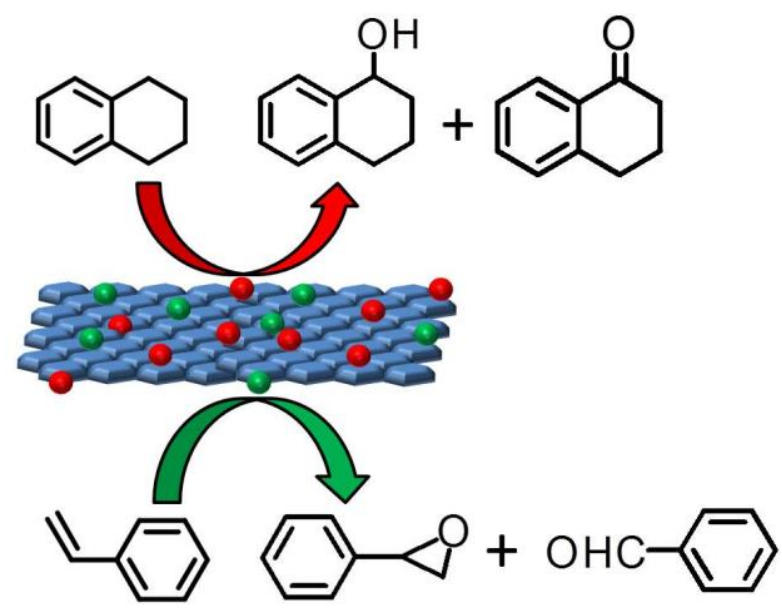


Scheme 10. Aerobic oxidation of tetraline and styrene promoted by $[B, N] G$.

One particular case of aerobic oxidation that is worth commenting to show the importance of the selection of the appropriate $\mathrm{G}$ material and also with respect the operating reaction mechanism is the aerobic oxidation of styrene [47]. In pioneering studies, Bielawsky reported that GO promotes polymerization of styrene, even in the presence of oxygen [64]. The presence of oxygen during styrene polymerization rules out the intermediacy of 1-phenylethyl radical in the process, since this radical will be quenched completely by molecular oxygen. Thus, the most probably styrene polymerization mechanism promoted by GO appears to derive from acid catalysis, due to the presence on $\mathrm{GO}$ of residual acidity, probably due to the presence of minor amounts of hydrogen sulfate groups [64]. In contrast, doped Gs obtained by pyrolysis of natural biopolymers are not acidic and they do not promote acid catalyzed polymerization. In the presence of oxygen, doped Gs promote autooxidation of styrene (Scheme 10) [47]. The reaction kinetics exhibits an induction period that probably reflects of the generation from styrene of the first $C$ centered radicals. One important observation was that the primary reaction product is benzaldehyde with selectivity at initial stages higher than $90 \%$. Benzaldehyde can arise from the oxidative cleavage of the $\mathrm{C}=\mathrm{C}$ bond of styrene. However, as reaction progresses, and styrene conversion increases growing the concentration of benzaldehyde, a change in the product distribution occurs and formation of styrene oxide in increasing selectivity is observed. For high styrene conversions, the selectivity towards styrene oxide can be over $50 \%$ and epoxide can become the major product, with a final yield higher than benzaldehyde. Figure 2 presents a time conversion plot for styrene oxidation, showing the induction period and the variations in the reaction selectivity. It should be commented that styrene oxide is the most wanted reaction product, while benzaldehyde has relatively low value, since it can be obtained from toluene in a more convenient way. Accordingly, it would be highly desirable to develop a catalyst for high selective alkene epoxidation, without leading to $\mathrm{C}=\mathrm{C}$ bond breaking. Selective oxidation of styrene and alkenes in general by molecular oxygen to epoxides is an important industrial process that still has to be developed to achieve high selectivity to the epoxide at high alkene conversion. 


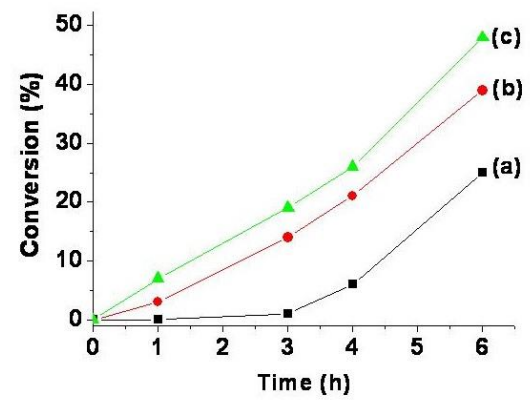

Figure 2. Time conversion plot for styrene oxidation upon initial addition of (a) $0 \%$, (b) $5 \%$ and (c) $10 \%$ of benzaldehyde.

The crossover in the product selectivity between benzaldehyde and styrene oxide was interpreted as mainly due to the involvement in styrene epoxidation of peroxyl radicals derived from benzaldehyde. Thus, at the initial stages of the reaction, in the absence of benzaldehyde no epoxidation can take place. In contrast, when benzaldehyde is present, benzoyl peroxyl radicals formed from benzaldehyde promote the aerobic styrene epoxidation. In support of this mechanistic proposal it was found that if styrene oxidation catalyzed by $G$ is carried out adding on purpose since the initial reaction time some benzaldehyde, the kinetics of the reaction changes. In the presence of benzaldehyde much shorter induction periods are observed and styrene oxide appears as primary product.

Aerobic oxidation of hydrocarbons, benzylic as well as cyclic or linear, can also be promoted by a combination of $G$ and graphitic carbon nitride $\left(g-C_{3} N_{4}\right)$ [65]. Comparison of the catalytic activity of the $\mathrm{G} / \mathrm{g}-\mathrm{C}_{3} \mathrm{~N}_{4}$ with that of the two components independently shows that there is a synergism between $G$ and g- $C_{3} N_{4}$. g- $C_{3} N_{4}$ can be obtained in different ways starting from melamine, urea and the isocyanine among other precursors [65]. $\mathrm{g}-\mathrm{C}_{3} \mathrm{~N}_{4}$ is a layered material having melon as building units resembling in a certain way the structure of $G$, but being different due to the regularity in the distribution of $\mathrm{N}$ atoms in the structure and the presence of holes among the not fully condensed melon units. Scheme 11 shows a model of the structure of $g-C_{3} N_{4}$. One of the consequences of the composition and structure of $\mathrm{g}_{-} \mathrm{C}_{3} \mathrm{~N}_{4}$, besides it easy preparation, is the stability of this material against oxidation. This catalysts stability is 
reflected by the fact the $\mathrm{G} / \mathrm{g}-\mathrm{C}_{3} \mathrm{~N}_{4}$ conjugate can be reused in these oxidation reactions for six consecutive runs without observing much decay on the catalytic activity [65].

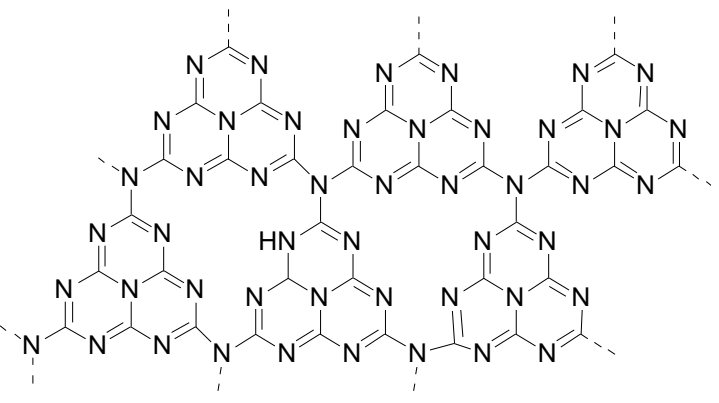

Scheme 11. Ideal structure of the $\mathrm{g}-\mathrm{C}_{3} \mathrm{~N}_{4}$ showing the melon units.

As commented in the section describing preparation procedures, one of the most common methods to obtain $G$ starts with the Hummers oxidation of graphite under harsh reaction conditions. By means of a detailed electron microscopy study it was found that the $\mathrm{r}-\mathrm{GO}$ resulting from this preparation procedure contains some holes on the $\mathrm{G}$ layer that have been observed to be frequently occupied by amorphous carbon debris, resulting from the aggressive chemical treatment of graphite. These amorphous carbon debris are acidic and r-GO can be purified from these amorphous carbon NPs by washing with aqueous base followed by a neutralization stage (Scheme 12). This acid-base washings procedure liberates the holes on $r-G O$ and these free holes can be highly efficient as oxidation centers. It has been reported that $\mathrm{r}$-GO devoid of debris by treatment with base and acids is a catalyst for the aerobic oxidation of benzylamines $[66,67]$.
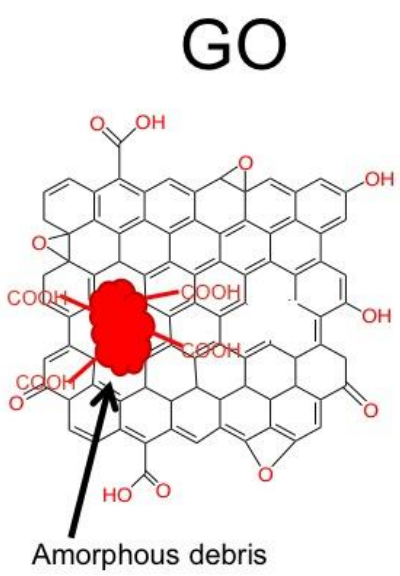

$\mathrm{r}-\mathrm{GO}$

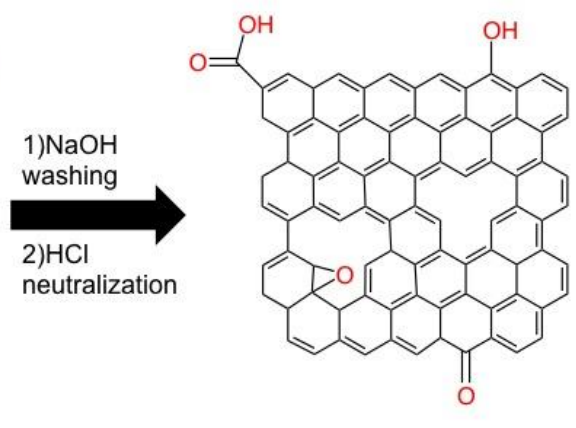


Scheme 12. Hummers oxidation of graphite under hash reaction conditions results in GO samples containing amorphous carbon debris. Holes can be freed from amorphous acid carbon debris by consecutive basic, acid washings.

Besides aerobic oxidation, doped $G$ can also be catalyst for the oxidation of organic compounds using peroxides. In one of these examples, benzylic hydrocarbons were oxidized by tert-butyl hydroperoxide (TBPH) using [N]G as catalyst in the presence of oxygen [68]. It was proposed that the active sites are the $\mathrm{N}$ atoms on $\mathrm{G}$ and the system could be reused five times without decay in the catalytic activity (Scheme 13) [68].

While organic hydroperoxides are adequate reagents for oxidations in organic solvents, peroxide monosulfate is one of the preferred reactive peroxides in water. Using phenol as probe of bio-reluctant organic pollutants, it has been reported that $[\mathrm{B}, \mathrm{N}] \mathrm{r}-\mathrm{GO}$ is a reliable catalyst that promotes phenol oxidation using peroxy monosulfate as oxidant.

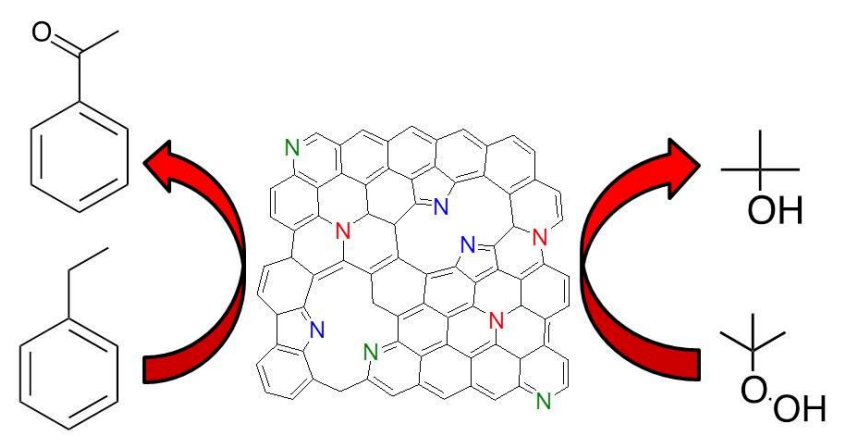

Scheme 13. Benzylic hydrocarbon oxidation by TBPH using [N]-G.

As it can be deduced from the above-comments, the preferred doped $G$ for most of the oxidation reactions contains $\mathrm{N}$. This could probably a reflection of the fact that the most studied sample as catalyst is N-doped G. It would be highly desirable to expand the screening of doped Gs and also to optimize the catalytic activity of $\mathrm{N}$ doped $\mathrm{G}$ by controlling the loading and distribution of this heteroatom. Co-doping with other heteroatoms is also a possibility to increase further the catalytic activity of doped Gs in oxidation reactions.

\section{Reduction reactions.}


While GO and doped Gs have been widely used as carbocatalysts for oxidation reactions, the use of graphenic materials for reductions has been comparatively less studied. In some occasions, theoretical calculations have predicted the catalytic activity of these materials before the experimental kinetic study has been actually performed. One of these interesting examples that we have already commented earlier is the theoretical calculation predicting that molecular hydrogen may undergo dissociative adsorption on the ribbon edges of doped $G$ [58]. The periphery of ideal $G$ models can be either armchair or zig-zag (Scheme 14). These terminal carbon atoms are ideal to interact with small substrates like molecular hydrogen due to their incomplete coordination.
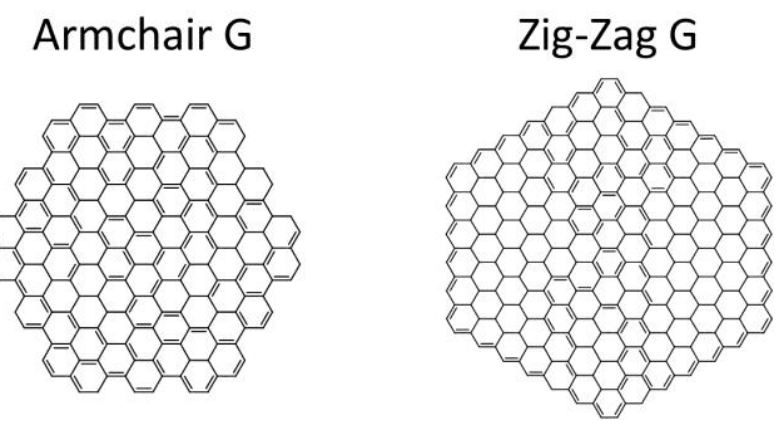

Scheme 14. Schematic illustration of $\mathrm{G}$ armchair (left) and zig-zag $\mathrm{G}$ edge.

These studies are certainly of interest in the context of developing G-based catalysts for hydrogenation reactions. Catalytic hydrogenations are one of the paradigmatic reactions that require noble metals to be performed. Thus, this reaction would be an important case of study to prove the concept that metal-free catalysts based on $\mathrm{G}$ materials can be of general applicability to promote any organic reaction. Active sites on the $\mathrm{G}$ sheet could be implemented by introducing the adequate dopant heteroatoms. Thus, it would be important to show that catalytic hydrogenations can be carried out promoted by $\mathrm{G}$ in the absence of any metal.

Besides catalytic hydrogenation of multiple $\mathrm{C}-\mathrm{C}$ bonds that would require some step of activation of molecular hydrogen, another type of reduction reactions are those involved in the conversion of $\mathrm{NO}_{x}$ to $\mathrm{N}_{2} . \mathrm{NO}_{x}$ gases include among other molecules $\mathrm{NO}, \mathrm{NO}_{2}$ and $\mathrm{N}_{2} \mathrm{O}$ and they constitute one of the most important contributors to atmospheric pollution, particularly in urban areas. $\mathrm{NO}_{\mathrm{x}}$ gases are 
generated by reaction of $\mathrm{N}_{2}$ and $\mathrm{O}_{2}$ present in air at temperatures above $1500{ }^{\circ} \mathrm{C}$. These conditions are typically encountered in combustion engines and burners and formation of $\mathrm{NO}_{x}$ gases is a consequence of the chemical composition of air and the equilibrium constants between $\mathrm{N}_{2}, \mathrm{O}_{2}$ and their corresponding $\mathrm{NO}_{x}$ products. Theoretical calculations at the DFT level predict that Si doping on G should increase the adsorption of $\mathrm{NO}_{x}$ gases and this $\mathrm{Si}$ doping should also implement catalytic activity on $\mathrm{G}$ to reduce $\mathrm{NO}_{x}[69,70]$. This type of doped $\mathrm{G}$ catalyst could have application for the treatment of exhaust gases in automotive muffles and in burners. As in the previously commented theoretical study on hydrogen activation, it would be a target in the area the preparation of $\mathrm{Si}$ doped $\mathrm{Gs}$ in order to evaluate their catalytic activity for $\mathrm{NO}_{\mathrm{x}}$ reductions. This is again a clear example of how theoretical modeling and computational calculations can be useful to lead the experimental work by predicting target materials to be synthesized and by indicating the structure of possible active sites for chemical reactions. Currently Cu-exchanged zeolites are among the most studied catalyst for $\mathrm{NO}_{\mathrm{x}}$ reductions [71] and it is important to establish if doped $\mathrm{G}$ can be equally effective for this reduction reaction of large importance in environmental chemistry.

There are many examples in the literature in which the reduction of 4nitrophenol to 4-aminophenol by $\mathrm{NaBH}_{4}$ has been performed in the presence of catalysts [72]. This reaction has also been one of the favorite transformations to compare the catalytic activity of different Gs. Among the reasons why the reduction of this probe molecule has been selected as a case of study, the most important ones are its simplicity and the fact that the process can be conveniently followed by UV-Vis absorption spectroscopy, monitoring the disappearance 4-nitrophenolate band at about $400 \mathrm{~nm}$ and monitoring the growth of the absorption band due to the reaction product at about $320 \mathrm{~nm}$. Moreover, when the reaction is carried out using a large excess of $\mathrm{NaBH}_{4}$, then, the kinetics becomes first order and the rate constant under these conditions can be used as a numerical value to rank the activity of different $G$ catalysts (Scheme 15). In this context, it has been found that N-doped G obtained from reduction of GO conveniently aminated is an efficient catalyst to promote 4nitrophenol reduction in the absence of metals. It was found that N-G can be reused 
up to 8 times and that there is not leaching of nitrogen from the catalyst to the solution [55]. Although the applied interest of this reaction type may be limited due to the high cost of $\mathrm{NaBH}_{4}$ compared to $\mathrm{H}_{2}$, it can serve to show that the process can be catalyzed in the absence of transition metals and that $\mathrm{N}$ atoms on $\mathrm{G}$ can act as active sites of the process. Moreover, by varying the distribution of $\mathrm{N}$ atoms among the different families, a linear correlation between graphenic nitrogen atoms (those $\mathrm{N}$ atoms replacing a $\mathrm{C}$ atom in an ideal $\mathrm{G}$ sheet) and the reaction rate constant was obtained [55]. This linear correlation was proposed as a firm evidence in support that only this type of nitrogen is the one catalytically relevant and that the other two types of $\mathrm{N}$ atoms detected by XPS, namely, pyridinic and pyrrolic, are not involved in the reaction mechanism.
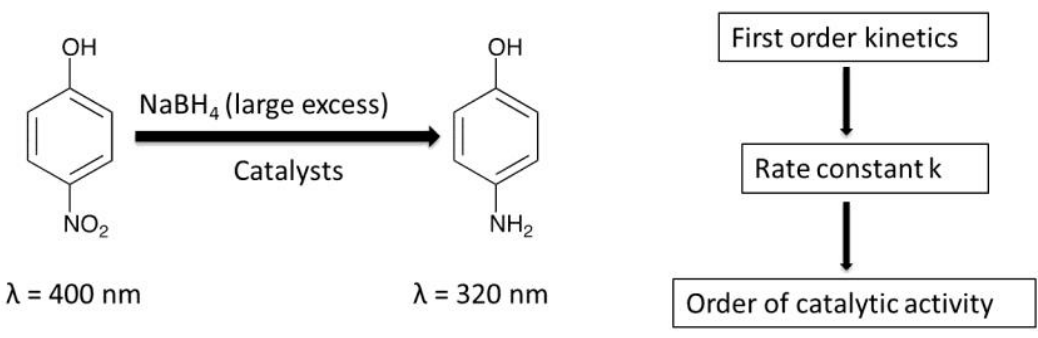

Scheme 15. 4-Nitrophenol reduction to 4-aminophenol using a large excess of $\mathrm{NaBH}_{4}$ and a catalyst as a procedure to rank the activity of different catalysts based on the rate constant value.

\section{Doped Gs as electrocatalyst.}

Due to the conductive nature of Gs these materials are among the favorite solids for the preparation of electrodes with special properties as sensors on catalyst [73]. Of particular relevance due to the potential application are the development of electrodes specially efficient for electrochemical oxygen reduction reactions (ORR) and oxygen evolution reaction (OER) since these processes are involved in fuel cells (Equations 1 and 2). 


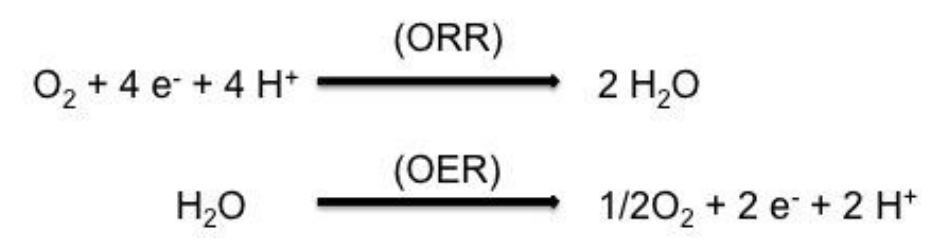

Equation 1 and 2. Equations of the electrochemical processes involved in fuel cells.

The operation of the fuel cell and the maximum power that can be obtained with them depend on how fast oxygen is reduced electrochemically. At the moment, the most convenient electrodes for ORR are based on platinum because electrodes of this metal exhibit the lowest overpotential for this reaction [73, 74]. However, commercial applications of fuel cells cannot be based on the massive use of platinum and other more affordable alternatives had to be considered. In this context, it has been found that $\mathrm{N}$-doped $\mathrm{G}$ can be considered as the last generation of ORR electrodes due to the excellent performance and durability of this doped $\mathrm{G}$ material for this type of reaction that under certain conditions can be close to that of platinum electrodes $[73,74]$. Similarly $\mathrm{N}$ doped $\mathrm{G}$ can be useful for OER to liberate oxygen from water that is a process related with electrocatalytic hydrogen generation from water. DFT calculations predict that substitution of carbon at $\mathrm{G}$ edges by nitrogen atoms should produce a material with the best OER and ORR performance in terms of overpotential that can be similar to those exhibited by platinum electrodes [73]. It has also been calculated that electron transfer from oxygen molecules to $\mathrm{N}$ doped $\mathrm{G}$ can take place through long distance without the need of oxygen adsorption on the $G$ sheet as a prerequisite [73, 74]. In addition it has also calculated that the potential of $\mathrm{N}$-doped $\mathrm{G}$ electrodes can be tuned by reducing the size of the $G$ sheet [73]. As we have already said these theoretical calculations have considerable importance to provide a conceptual framework to rationalize the behavior of different materials. Considering the vast amount of reports on the use of other doped $G$ as electrocatalysts the reader is referred to existing comprehensive reviews in this area for a complete coverage [7577].

\section{G as support of metal NPs in catalysis}


Metal and metal oxide particles of very small dimensions in nanometer scale are currently among the most studied catalysts [1-3]. There are abundant literature data showing that the catalytic activity of NPs depends on the particle size $[6,7]$, there being an interest in the control of the particle distribution during the preparation of the catalyst. One general observation in this research field is the growth of the NP size during the use of these materials as catalyst. To avoid agglomeration of NPs, increasing the stability of the catalyst under operation conditions, as well as to facilitate the recovery and reuse of NPs, one of the most widely used strategies is to deposit these NPs on large surface area supports.

As commented in introduction, 2D G-based materials offer considerable promise as supports for metal or metal oxide NPs due to the unique morphological and electronic properties of $\mathrm{G}$ that contribute positively to the stabilization of these NPs for their use as reusable and recoverable catalyst.

As for many other supports, noble metal NPs supported on Gs have been widely used to promote a large variety of reactions including oxidations, reductions and crosscouplings [78-81]. The preferred novel metals include $\mathrm{Au}, \mathrm{Pd}, \mathrm{Pt}, \mathrm{Ag}, \mathrm{Rh}$ and their alloys. Also, seminoble metals that can also be obtained predominantly in the 0 oxidation state such as $\mathrm{Ru}, \mathrm{Ni}$, and $\mathrm{Cu}$ have also been reported for oxidation and reduction reactions [82-84]. The most general procedure for the preparation of these G-supported NPs consist in adapting conventional reduction procedures for the preparation of NPs from suitable metal salts in the presence of $G$ materials or the addition of $\mathrm{G}$ supports on performed metal NPs. (Scheme 16).

\section{General preparation procedures}

$$
\begin{gathered}
\mathrm{M}^{N^{+}}+\mathrm{G} \frac{\mathrm{i}}{\mathrm{ii}} \mathrm{MNPs/G} \\
\text { i.- reduction } \\
\text { ii.- anchoring on } \mathrm{G} \\
\mathrm{M}^{\mathrm{N}^{+}} \stackrel{\mathrm{i}}{\longrightarrow} \mathrm{MNPs} \stackrel{\mathrm{ii}}{\longrightarrow} \mathrm{MNPs} / \mathrm{G}
\end{gathered}
$$

Scheme 16. Common methods for preparation of MNPs 
From the existing literature data there are, however, conflicting reports about whether or not the metal NPs detaches from the G-based material or if on the contrary they remain strongly anchored to the G support. It should be commented that typical preparation procedures for metal NPs (MNPs) such as the polyol method in which the MNPs are formed in ethylene glycol at high temperatures should lead to MNPs surrounded by molecules of the solvent or other additives present in the medium acting as ligands. The presence of these molecules on the surface of MNPS could be detrimental for the strong anchoring of the metal on the $G$ support. In other words care should be taken during the formation of MNPs to avoid the presence of strong ligands that could impede the interaction between MNPs and G.

In this context it has been frequently observed that the particle size of MNPs increases a few $\mathrm{nm}$ when these MNPs are supported on G compared to the size of the same MNPs as colloidal solution. This size increase can be taken as an evidence of the wetting of the surface of $G$ by MNPs that adapt their morphology for a better interaction with the $\mathrm{G}$ sheet. However, in other cases, a different strategy consisting in the addition of polymers or small ligands to increase the adhesion between the MNPs and $G$ has been reported $[82,85]$ (see Scheme 17).

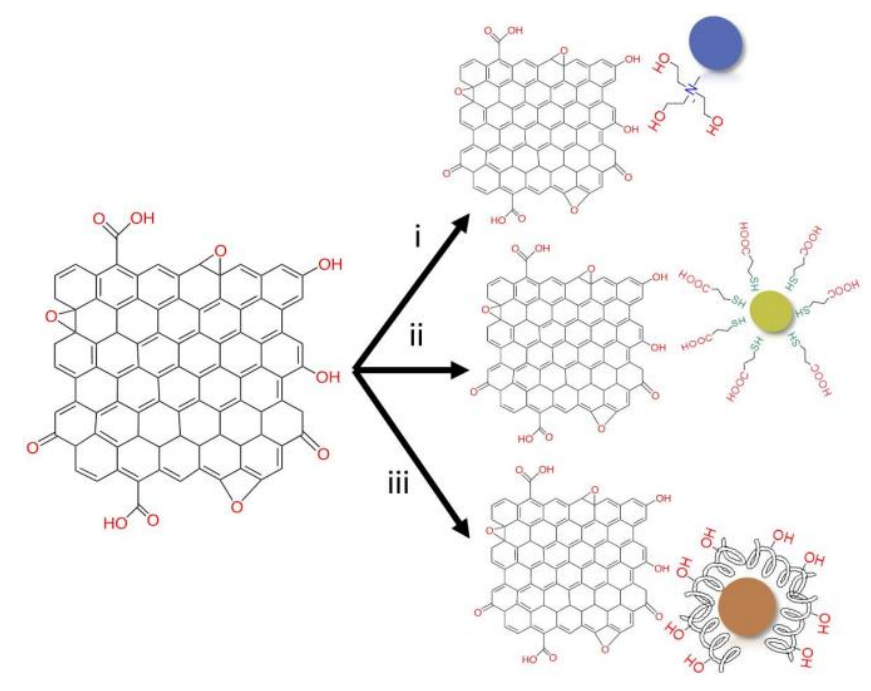

Scheme 17. Ways to increase adhesion between MNPs and $G$ by the presence of additives as interphase: (i) ionic liquid, (ii) small molecule or (iii) polymers.

In these examples the role of the polymers or small ligands is to act as interface between MNPs and Gs reducing the extent of leaching of the metal. Typical polymers 
that have been used for this purpose include polylysine, dextrans and dendrimers. Among the preferred small molecules, imidazolium ionic liquids, thiolated units and nitrogenated compounds have been reported $[85,86]$. As a general rule in this field, it has to be said that the presence of these additives should decrease the catalytic activity of MNPs by covering the metal surface and their use has to be justified by a reduction in the percentage of leaching to the point that this positive effect compensates the presumably lower activity and the more complex catalyst preparation.

Besides MNPs, metal oxides of transition metals have also been supported on $\mathrm{G}$ materials and used as catalysts in the aerobic oxidations of olefins and alcohols, low temperature $\mathrm{CO}$ oxidation and the $\mathrm{NO}_{\mathrm{x}}$ treatments, as well as Fischer-Tropsch process and reductions of multiple bonds using hydrazine as reagent.

The combination of MNPs and metal oxide NPs supported on G as catalyst has also been reported. In a number of examples the metal oxide was iron oxides with magnetic properties that have as main role to allow the easy recovery of the $G$ catalyst by magnetic fields. In these examples, the noble MNPS are the active sites for the process and $\mathrm{Fe}_{2} \mathrm{O}_{3}$ or other iron oxide phases are present without interfering in the catalytic activity but implementing a magnetic response on the material (Scheme 18).

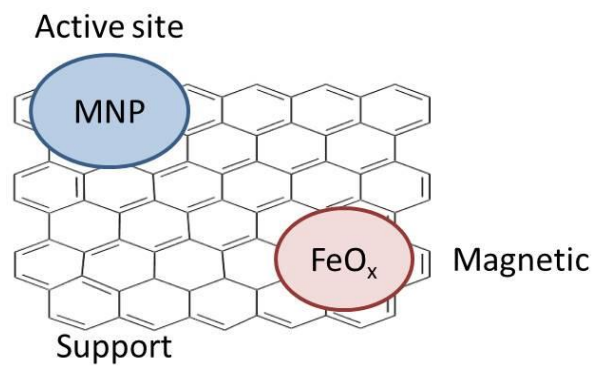

Scheme 18. Role of magnetic $\mathrm{FeO}_{\mathrm{x}} \mathrm{NPs}$ to prepare magnetoresponsive MNPs-G catalysts.

The large majority of reports in this field had used GO or r-GO as supports. It has been commented earlier that theoretical calculations suggest that the presence of dopant elements on $G$ should increase the interaction of the graphenic sheet with the NP. However, additional examples of the benefits of doping on $\mathrm{G}$ will be welcome to 
delineate the advantages of $G$ doping for this purpose. The important point is to establish the attachment of MNPs on the support without deterioration of the catalytic activity expected for MNPs. For example, DFT calculations indicate that B doping should be beneficial for increasing the activity of Fe NPs supported on G for the low temperature decomposition of ammonia to nitrogen and hydrogen [87].

Cross-coupling reactions catalyzed by Pd NPs have become among the most widely used processes in organic synthesis due to the general scope, high yields and mile conditions. Particularly, Suzuki-Miyaura coupling of aryl halides and aryl boronic acids to form asymmetric biphenyls is used in the preparation of specialty chemicals of high added value. In a pioneer work in this area Mülhaupt and co-workers found that Pd NPs supported on GO and Gs derived therefrom are extremely efficient catalyst for this type of reactions [88]. $G$ as support was found much more active than active carbon. As a follow up of the previous study it was found that Pd Nps obtained by the polyol method from $\mathrm{PdCl}_{2}$ supported on highly $\mathrm{N}$ doped $\mathrm{G}$ promote efficiently the SuzukiMiyaura cross-coupling and the Ullmman type reaction of a broad range of aryl halides in aqueous media, the activity of the material being attributed to the high nitrogen content of the $\mathrm{G}$ sheets. Pd supported on $\mathrm{N}$ doped $\mathrm{G}$ was a stable catalyst and can be reduced without a significant decrease I the catalytic activity [89].

In another work, Pd NPs supported on G or B-doped G have been used as catalysts for the dehydrogenative coupling of hydrosilanes and secondary amines (Scheme 19)[90]. $\mathrm{Pd}$ NPs were formed by the polyol method using $\mathrm{K}_{2} \mathrm{PdCl}_{4}$ as precursor.

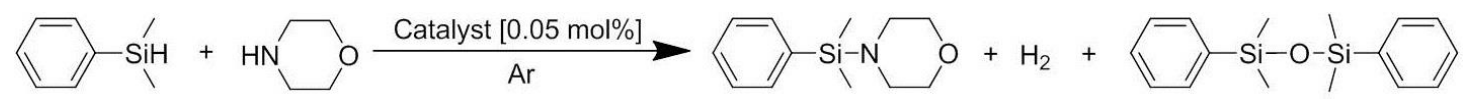

Scheme 19. Dehydrogenative coupling of dimehtylphenylsilane and morpholine in the presence of Pd NPs supported on G or doped G.

Figure 3 shows TEM and AFM images of the [Pd]G materials prepared as catalysts for this reaction. It was found that the presence of $\mathrm{N}$ as dopant decreases somewhat the catalytic activity for the reaction compare to B-doped G and G (see Table 1). 

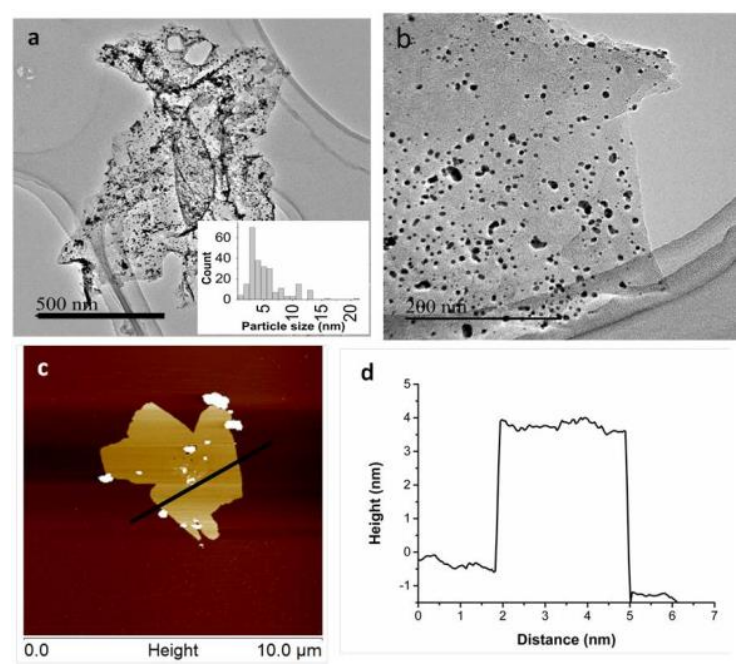

Figure 3. TEM images ( $a$ and $b$ ) of $P d / G$ at different magnifications. The inset shows the Pd particle size distribution. AFM image (c) of a layer of exfoliated Pd/G catalyst and its section profile (d).

Table 1 Catalytic activity of Pd NPs supported on different carbon materials.

\begin{tabular}{lcclll} 
Catalyst & $\mathrm{T}\left({ }^{\circ} \mathrm{C}\right)$ & $\mathrm{t}(\mathrm{h})$ & $\begin{array}{l}\text { Conversion } \\
(\%)\end{array}$ & $\begin{array}{l}\text { Selectivity } \\
(\%)\end{array}$ & $\begin{array}{l}\text { Yield } \\
(\%)\end{array}$ \\
\hline $1 \quad \mathrm{Pd} / \mathrm{G}$ & 120 & 0,5 & 99 & 89 & 89 \\
$2 \mathrm{Pd} /(\mathrm{N}) \mathrm{G}$ & 120 & 0,5 & 84 & 74 & 62 \\
$3 \mathrm{Pd} /(\mathrm{B}) \mathrm{G}$ & 120 & 0,5 & 96 & 87 & 84
\end{tabular}

Comparison with the catalytic activity of Pd NPs supported on [B]G show, however, that the performance of this catalyst based on $\mathrm{G}$ is similar to that of analogous Pd NPs supported on MWCNTs or even active carbon [90]. Thus apparently no advantage from the catalytic point of view is obtained by using doped G as support compared to CNTs. In contrasts to the case of Pd-[B]G, Cu NPs supported on [B]G are inactive as catalyst for the dehydrogenative coupling of silanes and amines [91]. However, $\mathrm{Cu}-[\mathrm{B}] \mathrm{G}$ is an efficient catalyst for analogous coupling between silanes and alcohols [91]. The failure of $\mathrm{Cu}$ NPs to promote the coupling of amines, in comparison with the successful coupling with alcohols, is not totally unexpected considering that $\mathrm{Cu}$ species have high affinity for amines that can be poisons of this metal for different reactions. In parallel with the behavior of the Pd NPs for the coupling between silanes and amines, also for Cu NPs supported on G as catalyst of the reaction between silanes and alcohols, it was 
found that $\mathrm{N}$-doped $\mathrm{G}$ is a less suitable support than $\mathrm{B}$-doped $\mathrm{G}$ that appears to be more active than undoped $G$ [91]. Figure 4 shows temporal profile of the dehydrogenative coupling promoted by a series of doped G-supported Cu NPs showing the influence that doping can exert on the performance of attached Cu NPs. For this reaction it was found that the catalyst can be reused with a gradual decrease in catalytic activity, probably due to $\mathrm{Cu}$ leaching as determined by chemical analysis of $\mathrm{Cu}$ in the supernatant solution after removal of the catalyst (Scheme 20 reaccion).

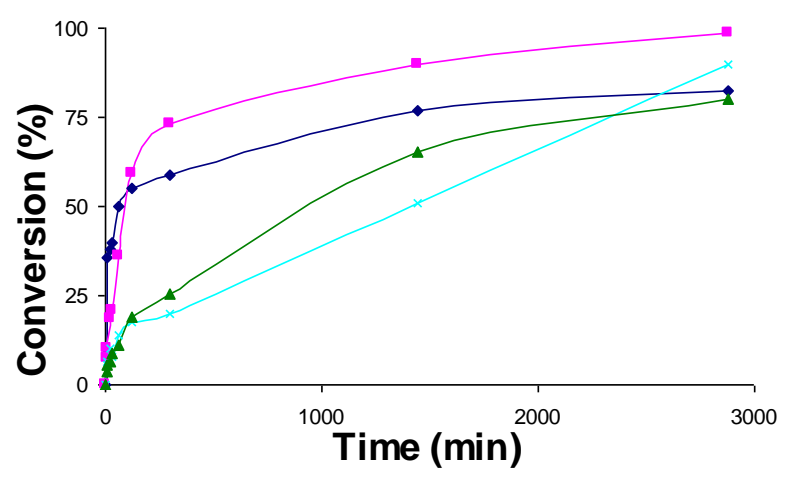

Figure 4. Conversion profile for the different catalyst Cu-G (black), Cu-[B]G (red), Cu$[\mathrm{B}, \mathrm{N}] \mathrm{G}$ (blue) and $\mathrm{Cu}-[\mathrm{N}] \mathrm{G}$ (green).

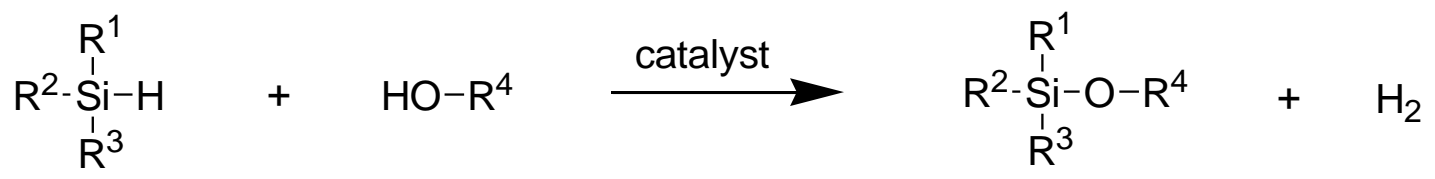

Scheme 20. Catalytic dehydrogenative o-silylation of alcohols by hydrosilanes.

A large percentage of metal oxide NPs supported on Gs have been used as catalysts for oxidation reactions. In one of these examples $\mathrm{Co}_{3} \mathrm{O}_{4} \mathrm{NPs}$ had been supported on an $\mathrm{r}-\mathrm{GO}$ doped with $\mathrm{N}$. The resulting $\mathrm{Co}_{3} \mathrm{O}_{4^{-}}[\mathrm{N}] \mathrm{r}-\mathrm{GO}$ exhibits catalytic activity for the oxidation of olefins and alcohols using $\mathrm{O}_{2}$ as terminal oxidant at moderate temperatures $\left(120^{\circ} \mathrm{C}\right)$ [92]. It was found, however, that the catalytic activity of the material decreases from 98 to $84 \%$ conversion upon reuse.

\section{Conclusions and future trends.}

The use of Gs as carbocatalysts although is developing at fast pace is still in its infancy due to the short period of time that has elapsed since the initial reports on the catalytic activity of these materials [14]. It can be easily predicted that the field will 
continue growing intensively and it will attract the interest of the catalytic community in the near future. Among the reasons for this interest in $G$ as catalysts, one of the most important is the fact that the cost and availability of some transition metals required as catalysts will grow and there are limited resources of these metals that may limit massive commercial applications in many cases. The main target in the area now is to delineate the scope of $\mathrm{G}$ as carbocatalyst. It is particularly important to know if $\mathrm{Gs}$ in the absence of any metal can promote those reactions that at the moment are considered that require metals to be promoted. The targets will be to show that Gs can be efficient catalysts for any oxidation or reduction reaction, as well as cross-couplings, including those that are based on organometallic catalysis. Theoretical models had to be developed simultaneously with the preparation of the required $G$ materials and evaluation of their catalytic activity, trying to determine the structure of suitable active sites and possible reaction mechanisms operating in the absence of metals. Besides theoretical models the use of in situ and in operando techniques should also provide crucial information on the reaction mechanism with the clear objective of establishing the type of reactions that can be catalyzed by Gs and the nature of the required active sites.

Also new and more convenient synthetic preparation protocols should be developed allowing the control not only of the dopant element present on $\mathrm{G}$ and its loading, but also the position of the dopant element on the sheet and its distribution among different families depending on the coordination and bonds.

Therefore, it can be easily anticipated that due to the intensive research effort and the combination of theoretical and experimental techniques, in a few years a more clear vision of the potential of Gs as heterogeneous catalysts will be obtained and, hopefully, many of the reactions that today are based on metal catalyst will be possibly performed using Gs. 


\section{References}

[1] M. Stratakis, H. Garcia, Chemical Reviews, 112 (2012) 4469-4506.

[2] R. Chinchilla, C. Nájera, Chemical Reviews, 107 (2007) 874-922.

[3] R. Jana, T.P. Pathak, M.S. Sigman, Chemical Reviews, 111 (2011) 1417-1492.

[4] A. Arcadi, Chemical Reviews, 108 (2008) 3266-3325.

[5] A.S.K. Hashmi, Chemical Reviews, 107 (2007) 3180-3211.

[6] D. Astruc, F. Lu, J.R. Aranzaes, Angewandte Chemie International Edition, 44 (2005) 7852 7872.

[7] A. Corma, H. Garcia, Chemical Society Reviews, 37 (2008) 2096-2126.

[8] N. Marion, S.P. Nolan, Chemical Society Reviews, 37 (2008) 1776-1782.

[9] A. Dhakshinamoorthy, S. Navalon, M. Alvaro, H. Garcia, ChemSusChem, 5 (2012) 46-64.

[10] A. Corma, H. Garcia, Advanced Synthesis \& Catalysis, 348 (2006) 1391-1412.

[11] S. Navalon, M. Alvaro, H. Garcia, Applied Catalysis B: Environmental, 99 (2010) 1-26.

[12] C. Baleizão, H. Garcia, Chemical Reviews, 106 (2006) 3987-4043.

[13] B.D. Sherry, A. Fürstner, Accounts of Chemical Research, 41 (2008) 1500-1511.

[14] D.R. Dreyer, C.W. Bielawski, Chemical Science, 2 (2011) 1233-1240.

[15] C. Su, K.P. Loh, Accounts of Chemical Research, 46 (2012) 2275-2285.

[16] J. Pyun, Angewandte Chemie International Edition, 50 (2011) 46-48.

[17] M.-M. Titirici, M. Antonietti, Chemical Society Reviews, 39 (2010) 103-116.

[18] T.-Y. Ma, L. Liu, Z.-Y. Yuan, Chemical Society Reviews, 42 (2013) 3977-4003.

[19] S. Navalon, A. Dhakshinamoorthy, M. Alvaro, H. Garcia, ChemSusChem, 4 (2011) 17121730.

[20] A. Schaetz, M. Zeltner, W.J. Stark, ACS Catalysis, 2 (2012) 1267-1284.

[21] D.S. Su, S. Perathoner, G. Centi, Chemical Reviews, 113 (2013) 5782-5816.

[22] G.G. Wildgoose, C.E. Banks, R.G. Compton, Small, 2 (2006) 182-193.

[23] H. Garcia, Advances in Chemistry, 2014 (2014) 20.

[24] K.S. Novoselov, A.K. Geim, S.V. Morozov, D. Jiang, Y. Zhang, S.V. Dubonos, I.V. Grigorieva, A.A. Firsov, Science, 306 (2004) 666-669.

[25] C.N.R. Rao, A.K. Sood, K.S. Subrahmanyam, A. Govindaraj, Angewandte Chemie International Edition, 48 (2009) 7752-7777.

[26] D.R. Dreyer, S. Park, C.W. Bielawski, R.S. Ruoff, Chemical Society Reviews, 39 (2010) 228240.

[27] X. Wang, X. Li, L. Zhang, Y. Yoon, P.K. Weber, H. Wang, J. Guo, H. Dai, Science, 324 (2009) 768-771.

[28] H. Wang, Y. Zhou, D. Wu, L. Liao, S. Zhao, H. Peng, Z. Liu, Small, 9 (2013) 1316-1320.

[29] R. Li, Z. Wei, X. Gou, W. Xu, RSC Advances, 3 (2013) 9978-9984.

[30] Z. Yang, Z. Yao, G. Li, G. Fang, H. Nie, Z. Liu, X. Zhou, X.a. Chen, S. Huang, ACS Nano, 6 (2011) 205-211.

[31] D. Chen, L. Tang, J. Li, Chemical Society Reviews, 39 (2010) 3157-3180.

[32] Q. Xiang, J. Yu, M. Jaroniec, Chemical Society Reviews, 41 (2012) 782-796.

[33] S. Navalon, A. Dhakshinamoorthy, M. Alvaro, H. Garcia, Chemical Reviews, 114 (2014) 6179-6212.

[34] X. Zhou, J. Qiao, L. Yang, J. Zhang, Advanced Energy Materials, 4 (2014) n/a-n/a.

[35] X.-K. Kong, C.-L. Chen, Q.-W. Chen, Chemical Society Reviews, 43 (2014) 2841-2857.

[36] N. Karousis, N. Tagmatarchis, D. Tasis, Chemical Reviews, 110 (2010) 5366-5397.

[37] P. Singh, S. Campidelli, S. Giordani, D. Bonifazi, A. Bianco, M. Prato, Chemical Society Reviews, 38 (2009) 2214-2230.

[38] K. Balasubramanian, M. Burghard, Small, 1 (2005) 180-192.

[39] S. Banerjee, T. Hemraj-Benny, S.S. Wong, Advanced Materials, 17 (2005) 17-29. 
[40] C. Su, M. Acik, K. Takai, J. Lu, S.-j. Hao, Y. Zheng, P. Wu, Q. Bao, T. Enoki, Y.J. Chabal, K. Ping Loh, Nat Commun, 3 (2012) 1298.

[41] J. Zhang, X. Liu, R. Blume, A. Zhang, R. Schlögl, D.S. Su, Science, 322 (2008) 73-77.

[42] S. Tang, Z. Cao, Physical Chemistry Chemical Physics, 14 (2012) 16558-16565.

[43] P. Wu, P. Du, H. Zhang, C. Cai, Physical Chemistry Chemical Physics, 15 (2013) 6920-6928.

[44] A.K. Geim, K.S. Novoselov, Nat Mater, 6 (2007) 183-191.

[45] Y. Xue, D. Yu, L. Dai, R. Wang, D. Li, A. Roy, F. Lu, H. Chen, Y. Liu, J. Qu, Physical Chemistry Chemical Physics, 15 (2013) 12220-12226.

[46] X. Duan, S. Indrawirawan, H. Sun, S. Wang, Catalysis Today.

[47] A. Dhakshinamoorthy, A. Primo, P. Concepcion, M. Alvaro, H. Garcia, Chemistry - A European Journal, 19 (2013) 7547-7554.

[48] S. Chauhan, P. Srivastava, A. Shrivastava, Appl Nanosci, 4 (2014) 461-467.

[49] D. Wei, Y. Liu, Y. Wang, H. Zhang, L. Huang, G. Yu, Nano Letters, 9 (2009) 1752-1758.

[50] A. Primo, P. Atienzar, E. Sanchez, J.M. Delgado, H. Garcia, Chemical Communications, 48 (2012) 9254-9256.

[51] P.-E. Gustavsson, P.-O. Son, Chapter 6 - Monolithic Polysaccharide Materials, in: T.B.T. František Švec, D. Zdeněk (Eds.) Journal of Chromatography Library, Elsevier, 2003, pp. 121141.

[52] M. Latorre-Sánchez, A. Primo, H. García, Angewandte Chemie International Edition, 52 (2013) 11813-11816.

[53] Copyright, in: H. Derek (Ed.) Advances in Carbohydrate Chemistry and Biochemistry, Academic Press, 2011, pp. iv.

[54] H. Wang, T. Maiyalagan, X. Wang, ACS Catalysis, 2 (2012) 781-794.

[55] X.-k. Kong, Q.-w. Chen, Z.-y. Lun, Journal of Materials Chemistry A, 2 (2014) 610-613.

[56] X.-k. Kong, Z.-y. Sun, M. Chen, C.-I. Chen, Q.-w. Chen, Energy \& Environmental Science, 6 (2013) 3260-3266.

[57] Y. Gao, D. Ma, C. Wang, J. Guan, X. Bao, Chemical Communications, 47 (2011) 2432-2434.

[58] T. Liao, C. Sun, Z. Sun, A. Du, S. Smith, Physical Chemistry Chemical Physics, 15 (2013) 8054-8057.

[59] A. Primo, F. Neatu, M. Florea, V. Parvulescu, H. Garcia, Nat Commun, 5 (2014).

[60] P. Wu, Y. Qian, P. Du, H. Zhang, C. Cai, Journal of Materials Chemistry, 22 (2012) 64026412.

[61] B. Das, B. Choudhury, A. Gomathi, A.K. Manna, S.K. Pati, C.N.R. Rao, ChemPhysChem, 12 (2011) 937-943.

[62] P. Lazar, S. Zhang, K. Šafářová, Q. Li, J.P. Froning, J. Granatier, P. Hobza, R. Zbořil, F. Besenbacher, M. Dong, M. Otyepka, ACS Nano, 7 (2013) 1646-1651.

[63] J. Long, X. Xie, J. Xu, Q. Gu, L. Chen, X. Wang, ACS Catalysis, 2 (2012) 622-631.

[64] D.R. Dreyer, H.-P. Jia, C.W. Bielawski, Angewandte Chemie International Edition, 49 (2010) 6813-6816.

[65] X.-H. Li, J.-S. Chen, X. Wang, J. Sun, M. Antonietti, Journal of the American Chemical Society, 133 (2011) 8074-8077.

[66] X.-H. Li, M. Antonietti, Angewandte Chemie International Edition, 52 (2013) 4572-4576.

[67] W.S. Hummers, R.E. Offeman, Journal of the American Chemical Society, 80 (1958) 13391339.

[68] Y. Gao, G. Hu, J. Zhong, Z. Shi, Y. Zhu, D.S. Su, J. Wang, X. Bao, D. Ma, Angewandte Chemie International Edition, 52 (2013) 2109-2113.

[69] Y. Chen, B. Gao, J.-X. Zhao, Q.-H. Cai, H.-G. Fu, J Mol Model, 18 (2012) 2043-2054.

[70] Y. Chen, Y.-j. Liu, H.-x. Wang, J.-x. Zhao, Q.-h. Cai, X.-z. Wang, Y.-h. Ding, ACS Applied Materials \& Interfaces, 5 (2013) 5994-6000.

[71] X. Yang, Z. Wu, M. Moses-Debusk, D.R. Mullins, S.M. Mahurin, R.A. Geiger, M. Kidder, C.K. Narula, The Journal of Physical Chemistry C, 116 (2012) 23322-23331. 
[72] K. Kuroda, T. Ishida, M. Haruta, Journal of Molecular Catalysis A: Chemical, 298 (2009) 711.

[73] K. Gong, F. Du, Z. Xia, M. Durstock, L. Dai, Science, 323 (2009) 760-764.

[74] Y. Sun, C. Li, G. Shi, Journal of Materials Chemistry, 22 (2012) 12810-12816.

[75] W.J. Lee, U.N. Maiti, J.M. Lee, J. Lim, T.H. Han, S.O. Kim, Chemical Communications, 50 (2014) 6818-6830.

[76] N. Daems, X. Sheng, I.F.J. Vankelecom, P.P. Pescarmona, Journal of Materials Chemistry A, 2 (2014) 4085-4110.

[77] Y. Zheng, Y. Jiao, M. Jaroniec, Y. Jin, S.Z. Qiao, Small, 8 (2012) 3550-3566.

[78] G. Wu, X. Wang, N. Guan, L. Li, Applied Catalysis B: Environmental, 136-137 (2013) 177185.

[79] N. Morimoto, S.-i. Yamamoto, Y. Takeuchi, Y. Nishina, RSC Advances, 3 (2013) 1560815612.

[80] T. Wu, L. Zhang, J. Gao, Y. Liu, C. Gao, J. Yan, Journal of Materials Chemistry A, 1 (2013) 7384-7390.

[81] Y. Nishina, J. Miyata, R. Kawai, K. Gotoh, RSC Advances, 2 (2012) 9380-9382.

[82] H.-W. Hu, J.H. Xin, H. Hu, ChemPlusChem, 78 (2013) 1483-1490.

[83] Ö. Metin, S. Ho, C. Alp, H. Can, M. Mankin, M. Gültekin, M. Chi, S. Sun, Nano Res., 6 (2013) 10-18.

[84] X. Liu, C. Meng, Y. Han, Nanoscale, 4 (2012) 2288-2295.

[85] R.K. Pandey, M.D. Hossain, S. Moriyama, M. Higuchi, Journal of Materials Chemistry A, 1 (2013) 9016-9018.

[86] W. Xiao, Z. Sun, S. Chen, H. Zhang, Y. Zhao, C. Huang, Z. Liu, RSC Advances, 2 (2012) 81898193.

[87] X. Liu, C. Meng, Y. Han, Physical Chemistry Chemical Physics, 14 (2012) 15036-15045.

[88] G.M. Scheuermann, L. Rumi, P. Steurer, W. Bannwarth, R. Mülhaupt, Journal of the American Chemical Society, 131 (2009) 8262-8270.

[89] S.K. Movahed, M. Dabiri, A. Bazgir, Applied Catalysis A: General, 488 (2014) 265-274.

[90] J.F. Blandez, I. Estebe-Adell, M. Alvaro, H. García, Chemical Communications, Submitted (2014).

[91] J.F. Blandez, A. Primo, A.M. Asiri, M. Álvaro, H. García, Angewandte Chemie International Edition, 53 (2014) 12581-12586.

[92] R. Nie, J. Shi, W. Du, W. Ning, Z. Hou, F.-S. Xiao, Journal of Materials Chemistry A, 1 (2013) 9037-9045. 